Understanding and suppressing the near Scrape-Off Layer heat flux feature in inboard-limited plasmas in TCV

This content has been downloaded from IOPscience. Please scroll down to see the full text.

Download details:

IP Address: 128.179.255.169

This content was downloaded on 14/08/2017 at 09:28

Manuscript version: Accepted Manuscript

Nespoli et al

To cite this article before publication: Nespoli et al, 2017, Nucl. Fusion, at press:

https://doi.org/10.1088/1741-4326/aa84e0

This Accepted Manuscript is: @ 2017 Ecole Polytechnique Federale de Lausanne

During the embargo period (the 12 month period from the publication of the Version of Record of this article), the Accepted Manuscript is fully protected by copyright and cannot be reused or reposted elsewhere.

As the Version of Record of this article is going to be / has been published on a subscription basis, this Accepted Manuscript is available for reuse under a CC BY-NC-ND 3.0 licence after the 12 month embargo period.

After the embargo period, everyone is permitted to copy and redistribute this article for non-commercial purposes only, provided that they adhere to all the terms of the licence https://creativecommons.org/licences/by-nc-nd/3.0

Although reasonable endeavours have been taken to obtain all necessary permissions from third parties to include their copyrighted content within this article, their full citation and copyright line may not be present in this Accepted Manuscript version. Before using any content from this article, please refer to the Version of Record on IOPscience once published for full citation and copyright details, as permission will likely be required. All third party content is fully copyright protected, unless specifically stated otherwise in the figure caption in the Version of Record.

When available, you can view the Version of Record for this article at: http://iopscience.iop.org/article/10.1088/1741-4326/aa84e0 


\section{Understanding and suppressing the near Scrape-Off Layer heat flux feature in inboard-limited plasmas in TCV}

F. Nespoli ${ }^{1}$, B. Labit ${ }^{1}$, I. Furno ${ }^{1}$, J. Horacek ${ }^{2}$, C.K. Tsui ${ }^{1,3}$, J.A. Boedo ${ }^{3}$, R. Maurizio ${ }^{1}$ H. Reimerdes ${ }^{1}$, C. Theiler ${ }^{1}$, P. Ricci ${ }^{1}$, F.D. Halpern ${ }^{1,4}$, U. Sheikh ${ }^{1}$, K. Verhaegh ${ }^{1}$ R.A. Pitts ${ }^{5}$, F. Militello ${ }^{6}$, the EUROfusion MST1 team ${ }^{7}$, the TCV team ${ }^{8}$

${ }^{1}$ Ecole Polytechnique Fédérale de Lausanne (EPFL), Swiss Plasma Center (SPC), CH-1015 Lausanne, Switzerland

${ }^{2}$ Institute of Plasma Physics, ASCR v.v.i., Za Slovankou 3, 18200 Prague, Czech Republic

${ }^{3}$ University of California-San Diego, La Jolla, California 92093, USA

${ }^{4}$ General Atomics, P.O. Box 85608, San Diego, California 92186-9784

${ }^{5}$ ITER Organization, Route de Vinon-sur-Verdon CS 90 046, F-13067 St Paul lez Durance Cedex, France

${ }^{6}$ CCFE, Culham Science Centre, Abingdon, Oxon, OX14 3DB, UK

${ }^{7}$ See author list of H. Meyer et al 2017 Nucl. Fusion 57102014

${ }^{8}$ See author list of S. Coda et al 2017 Nucl. Fusion 57102011

Email: nespolifederico@gmail.com

\section{Abstract}

In inboard-limited plasmas, the Scrape-Off Layer (SOL) shows two regions: the near SOL, extending a few $\mathrm{mm}$ from the Last Closed Flux Surface (LCFS), characterized by a steep gradient of the parallel heat flux radial profile, and a far SOL, typically some $\mathrm{cm}$ wide, with flatter heat flux profiles. The physics of the near SOL is investigated in TCV with two series of experiments featuring deuterium and helium plasmas, in which the plasma current, density and elongation have been varied. The parallel heat flux profiles are measured on the limiter by means of infrared thermography. For the first time, the near SOL is reported to disappear for low plasma current or at high density, for values of the SOL collisionality $\nu_{S O L}^{*}$ corresponding to a conduction-limited regime. The power in the near SOL $\triangle P_{S O L}$ is shown to decrease with the normalized Spitzer resistivity $\nu$ as $\Delta P_{S O L} \propto \nu^{-1}$. The floating potential profiles, measured at the limiter using flush-mounted Langmuir probes (LP), show the presence of non-ambipolar currents, and their relation to the presence of a velocity shear layer is discussed. The shearing rate is shown to strictly correlate with the power in the near SOL $\Delta P_{S O L}$, consistently with a recent theoretical model. Measurements of the near SOL on the Low Field Side (LFS) are performed using a reciprocating Langmuir probe (RP). The near SOL is reported to vanish simultaneously at the LFS and at the limiter. The near and far SOL widths are compared with the predictions from existing theoretical models, to which empirical corrections with resistivity and elongation are proposed.

\section{Introduction}

In magnetic confinement fusion devices, the open-field line region called Scrape-Off Layer (SOL) couples the "core" confined plasma with the solid surfaces of the reactor wall, affecting the plasma performances. Furthermore, the elevated thermal load resulting from the deposition of the SOL plasmas on the plasma facing components is considered one of the crucial problems to solve for fusion. Inboard limited L-mode plasmas are foreseen for future fusion reactors start-up and rampdown phases. In ITER, the plasma will be limited on the central column [1], which will be covered by beryllium (Be) tiles. In the standard model of the SOL of a limited plasma [2], the parallel heat 
flux radial profile in the SOL is described by an exponential radial decay:

$$
q_{\|}\left(r_{u}\right)=q_{0} \exp \left(-r_{u} / \lambda_{q}\right)
$$

where $r_{u}$ is the upstream radial coordinate at the outer midplane, $r_{u}=0$ at the Last Closed Flux Surface (LCFS), $\lambda_{q}$ is the heat flux decay length and $q_{0}$ is the parallel heat flux at the LCFS. However, recent infrared (IR) thermography and Langmuir probes (LPs) measurements of inboardlimited L-mode plasmas in many tokamaks $[3,4,5,6,7]$ have shown the ubiquitous presence of two regions in the SOL: a "near" SOL, extending typically a few $\mathrm{mm}$ from the LCFS, characterized by a steep gradient of the parallel heat flux, and a "far" SOL, typically a few cm wide, featuring flatter heat flux profiles. The parallel heat flux radial profile in the SOL is therefore better described by a sum of two exponentials

$$
q_{\|}\left(r_{u}\right)=q_{n} \exp \left(-r_{u} / \lambda_{n}\right)+q_{f} \exp \left(-r_{u} / \lambda_{f}\right)
$$

where $\lambda_{n}, \lambda_{f}$ are the parallel heat flux decay length in the near and far SOL, respectively, and $q_{n}$ and $q_{f}$ are the associated parallel heat flux magnitudes.

Even though a separation between near and far SOL is sometimes observed also in diverted configuration $[8,9]$, the ubiquitous presence of a near SOL in limited plasmas has recently attracted the attention of the fusion community. Indeed, the near SOL is responsible for the peak heat loads on the limiter, which can be a factor of 6 higher [10] than the predictions from Eq. (1). This can lead to severe damages to the limiter, as observed in JET [3]. The design of ITER First Wall (FW) panels has been changed to handle the heat flux associated with the near SOL [10]. Still, the physics governing the formation of the near SOL is not fully understood. Furthermore, helium (He) plasmas are foreseen for the ITER non-activation phase [11]. To date, no investigation of the near SOL had been performed in He plasmas.

Indeed, to improve our understanding of the near SOL, further experiments featuring both D and He plasmas have been performed in TCV, taking advantage of an improved and extended set of diagnostics, and leveraging the experience acquired in the previous experiments exposed in Ref. [4]. These experiments have been performed within the Medium-Size Tokamak (MST) Task Force of the EUROfusion consortium [12,13].

This paper is structured as follows: in section 2, the experimental setup and the database are presented. In section 3 , the methodology used to analyze the IR data is detailed. The perpendicular heat fluxes are described in section 4, while the parallel heat fluxes are discussed in section 5 , showing the presence of the near SOL in all discharges, except for low current and high density. The observed trends on the main plasma parameters are discussed. In section 6 , the near and far SOL widths are compared with existing scalings, for which some corrections are suggested. In section 7, the dependence of the power in the near SOL $\triangle P_{S O L}$ upon the normalized Spitzer resistivity $\nu$ and the SOL collisionality $\nu_{S O L}^{*}$ is investigated. In section 8 , we discuss the measurements of nonambipolar currents flowing to the limiter using flush-mounted LPs, and their correlation with the power in the near SOL. An interpretation involving poloidal velocity shear is provided. In section 9, we compare the profiles of parallel heat fluxes and floating potential measured at the High Field Side (HFS), with the ones measured at the Low Field Side (LFS) using a Reciprocating Probe (RP). Finally, in section 10, the main results of this paper are summarized.

\section{Experimental setup and experiments overview}

A dedicated set of experiments has been performed in the TCV tokamak at EPFL, Lausanne, Switzerland $[15,13]$. TCV is a medium sized tokamak $\left(R_{0} \approx 0.88 \mathrm{~m}, B_{0} \approx 1.44 \mathrm{~T}\right)$ with unique 
shaping capabilities due to 16 independently powered poloidal field coils and an open vessel structure. The TCV vacuum vessel is almost entirely covered with graphite protection tiles. The main diagnostics used in the experiments are: the horizontal infrared camera (HIR) system, an array of flush-mounted Langmuir probes (LP) embedded in the limiter, the newly installed reciprocating Langmuir probe (RP), on loan from UCSD [16]. The location of the diagnostics is shown in Fig. 1, together with the time evolution of the main plasma parameters and the magnetic equilibrium for the reference discharge.

The central piece of the HIR system [17] is an IRCam EQUUS $81 \mathrm{k}$ M fast framing, camera. Its detector is composed by $320 \times 256 \mathrm{CdHgTe}$ pixels, sensitive to mid-wave IR spectrum, nominally to photons with wavelength $3.7 \pm 0.2 \mu \mathrm{m}<\lambda<4.8 \pm 0.2 \mu \mathrm{m}$. In the present experiments, a long wavelength pass filter with cutoff at $4034 \mathrm{~nm}$ has been used, limiting the detector sensitivity to the range $4 \mu \mathrm{m}<\lambda<4.8 \mu \mathrm{m}$ to avoid the detector saturation and the short wavelength IR part of the spectrum, dominated by molecular emission [18]. The acquisition rate is $200 \mathrm{fps}$ and the integration time $t_{\text {int }}$ is $1.5 \mathrm{~ms}$. The HIR system monitors the temperature of the central column (CC) graphite tiles, acting as a limiter, to compute the heat flux deposited onto them. The HIR field of view (FOV), shown in Fig. 1e with red dashed lines, has been doubled by using wide angle optics, resulting in a spatial resolution of $1.6 \mathrm{~mm} / \mathrm{px}$.

The TCV CC is equipped with an array of flush-mounted LPs (orange dots in Fig. 1e). The probe

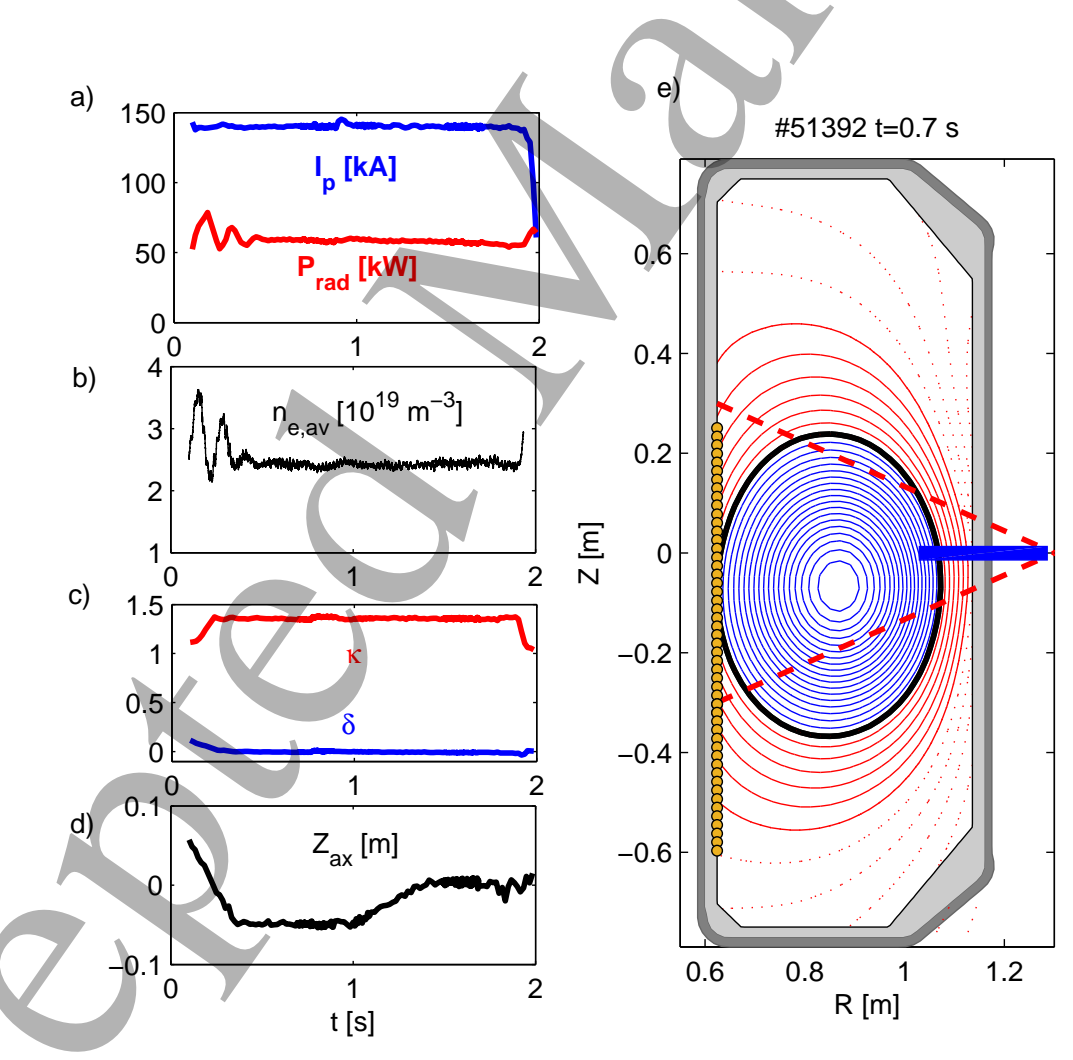

Figure 1: Time traces for discharge \#51392 of: a) plasma current $I_{p}$ (blue) and total radiated power $P_{\text {rad }}($ red), b) plasma average density , c) plasma elongation $\kappa$ (red) and triangularity $\delta$ (blue), d) vertical position of the magnetic axis $Z_{a x}$. e) Magnetic equilibrium reconstruction from LIUQE [14], together with the array of LPs (orange dots), the field of view of the HIR system (red dashed lines) and the RP trajectory (thick blue line). 
spacing is $17.2 \mathrm{~mm}$, and their cross section is circular with radius $r_{p}=2 \mathrm{~mm}$. In the experiments presented herein, the LPs monitored the ion saturation current $I_{s a t}$ and the floating potential $V_{f l}$. Some of the discharges in the database were repeated with the probes in sweeping mode (LPs biased with a swept potential) or measuring the current flowing to the (unbiased) limiter, $I_{0}$.

The newly installed RP, detailed in Ref. [16] can perform up to two reciprocations within a plasma discharge, covering the distance of $20 \mathrm{~cm}$ in $200 \mathrm{~ms}$. The radial projection of the probe trajectory is shown with a thick blue line in Fig. 1e. The RP is located on TCV middle port and the reciprocation takes place at the plasma outer midplane (OMP). The probe head assembly features 10 pins. In particular, for the experiments discussed here, two pins constitute a double probe monitoring the plasma temperature $T_{e}$ and ion saturation current density $J_{s a t}$, and one pin measures the floating potential $V_{f l}$ and its fluctuations.

A typical discharge is designed with three phases, where all the plasma parameters are kept constant (Fig. 1a-c), except the plasma vertical position $Z_{a x}$, which is varied in time, as shown in Fig. 1d, to ensure the following:

1. For $0.5 \mathrm{~s}<t<1 \mathrm{~s}, Z_{a x}=-7 \mathrm{~cm}$. This position optimizes the HIR FOV, since the plasma contact point is not near the tile edges or other regions that need to be excluded from the analysis (see Sec. 3 for details). Also, this position increases the range of the radial upstream coordinate $r_{u}$ entering the FOV, with respect to the case $Z_{a x}=0$.

2. For $1 \mathrm{~s}<t<1.2 \mathrm{~s}$ the vertical position is swept to increase the spatial resolution of the profiles measured with the LPs.

3. For $1.2 \mathrm{~s}<t<2 \mathrm{~s}, Z_{a x}=0$. This position is optimized for the RP plunging, ensuring that the reciprocation of the probe takes place at the OMP.

Two series of inboard limited L-mode deuterium (D) and helium (He) plasmas have been investigated, for a total of 36 discharges. For both species, systematic scans of plasma current $85 \leq I_{p}[\mathrm{kA}] \leq 210$, density $1 \leq n_{e, a v}\left[10^{19} \mathrm{~m}^{-3}\right] \leq 5$, and elongation $1 \leq \kappa \leq 1.5$, have been performed. The meeting point of the three scans is discharge \#51392 (shown in Fig. 1), with $I_{p}=140$ $\mathrm{kA}, n_{e, a v}=2.5 \cdot 10^{19} \mathrm{~m}^{-3}, \kappa=1.4$. For all the discharges the triangularity is $\delta=0$.

\section{Infrared data analysis method}

The heat fluxes deposited on the limiter are computed from the IR images using the THEODOR code [19], with a procedure similar to the one described in Ref. [4], and summarized in the following. The IR images are converted to temperature via an in-situ calibration performed with a heated tile, whose temperature is monitored by thermocouples. A series of corrections are applied in order to compensate for the effects of the strong magnetic field $(\sim 1 \mathrm{~T})$ on the camera detector, the vignetting, the barrel/distortion introduced by the wide angle optics, and the perspective deformation. Two tiles (one located at the equatorial plane of the device, $Z=0$, and the one above) are remapped onto the physical coordinates $(R \phi, Z)$, with $R$ the major radius coordinate and $\phi$ the toroidal angle (as shown in Fig. 2a), and used for the following analysis. THEODOR solves the heat diffusion equation only in two dimensions (in the direction perpendicular to the tile surface, and one direction parallel to the tile surface). Assuming that the heat diffuses mainly in the depth of the tiles, these are subdivided into 50 horizontal slices on which the deposited heat flux $q_{d e p}$ is computed. This assumption has been verified a posteriori, comparing the code results obtained for horizontal and vertical slices, and artificially suppressing the heat conductivity in the direction parallel to the tile surface, not resulting in substantial changes in the final $q_{d e p}$. The 50 
Figure 2: a) Deposited heat flux $q_{d e p}(R \phi, Z)$ obtained from THEODOR using 50 horizontal slices, together with the technical drawing of a tile and the set of coordinates. b) Radial upstream coordinate $r_{u}$. c) Angle between the magnetic field lines and the plane tangent to the tile surface $\alpha$. The line $\alpha=0$ is shown with blue dots. The blue crosses are the maximum of $q_{d e p}$ on each horizontal slice $(Z=$ const $)$. d) $q_{\text {dep }}$ selected for the following analysis.

slices are reassembled to obtain the temporal evolution of the 2D map of the heat deposited onto the tiles $q_{d e p}(R \phi, Z, t)$. The deposited heat flux is averaged over time in intervals where all plasma parameters, including the plasma vertical position $Z_{a x}$, are kept constant. The resulting $q_{d e p}(R \phi, Z)$ $2 \mathrm{D}$ map is shown in Fig. 2a.

The integration of the $q_{d e p}$ 2D map over the considered tile surface, corresponding to the upper side of the limiter, provides the power deposited on the CC, $P_{d e p}=32 \iint 2 q_{d e p} \mathrm{~d} R \phi \mathrm{d} Z$, where the factor 2 accounts for heat deposition on the lower side of the limiter, assumed to be symmetric for simplicity, and 32 is the number of tiles in the toroidal direction. For the here presented database, $P_{d e p}$, together with the total radiated power $P_{r a d}$ obtained from bolometric measurements, accounts on average for the $77 \%$ of the ohmic power $P_{\Omega}$. The power missing to complete the power balance could be partly transfered to the neutrals, and/or lost to the outer wall. Also, the TCV "naked" metal foil bolometers could provide an underestimation of the radiated power [20]. Asymmetry in the heat deposition in between the ion and electron drift side of the limiter, investigated in section 5 , could also play a minor role.

To compare discharges with different plasma shapes and parameters, we remap each point of the tiles onto magnetic coordinates, namely the upstream radial coordinate $r_{u}$ and the angle between the magnetic field and the plane tangent to the tile surface $\alpha$. The mapping is based on the magnetic equilibrium reconstruction by the LIUQE code [14], and the result is shown in Fig. 2. The TCV tiles are shaped in the toroidal direction to spread more uniformly the heat loads and to avoid exposed edges, the toroidal contour of the tile being composed of a cylindrical segment chamfered by elliptic edges [21]. This results in a variation of the angle $\alpha$ both along $R \phi$ and $Z$. In particular, for limited plasmas, a curve on the tiles for which $\alpha=0$ can be identified. This is shown by the blue dots in Fig 2b,c. 
Several zones are excluded from the subsequent analysis: the zone where the TCV port is imaged, the region shadowed by the neighboring tile, the regions where the tile thickness is much smaller than the average tile thickness (THEODOR assumes a constant tile thickness), the horizontal gap between the two tiles.

The resulting 2D map of deposited heat flux $q_{\text {dep }}\left(r_{u}, \alpha\right)$, shown in Fig. 2d, is used to compute the perpendicular and parallel components of the heat flux. The deposited heat flux is modeled as the sum of components parallel and perpendicular to the magnetic field, and a background component:

$$
q_{d e p}\left(r_{u}, \alpha\right)=q_{\|}\left(r_{u}\right) \sin \alpha+q_{\perp}\left(r_{u}\right) \cos \alpha+q_{B G} .
$$

The background heat flux $q_{B G}$ results from a combination of different processes like IR reflections, heating of the tiles by the radiation coming from the plasma, and IR radiation from runaway electrons, and accounts typically for the $\sim 1-5 \%$ of the peak heat load. The specific shape of the TCV tiles [21], shown in Fig. 2a, featuring a line of tangential incidence for limited plasmas, allows to estimate the cross-field heat flux at the limiter $q_{\perp}$. Indeed, for $\alpha=0$, the projection of the parallel heat flux vanishes so that

$$
q_{d e p}\left(r_{u}, \alpha=0\right)=q_{\perp}\left(r_{u}\right)+q_{B G}
$$
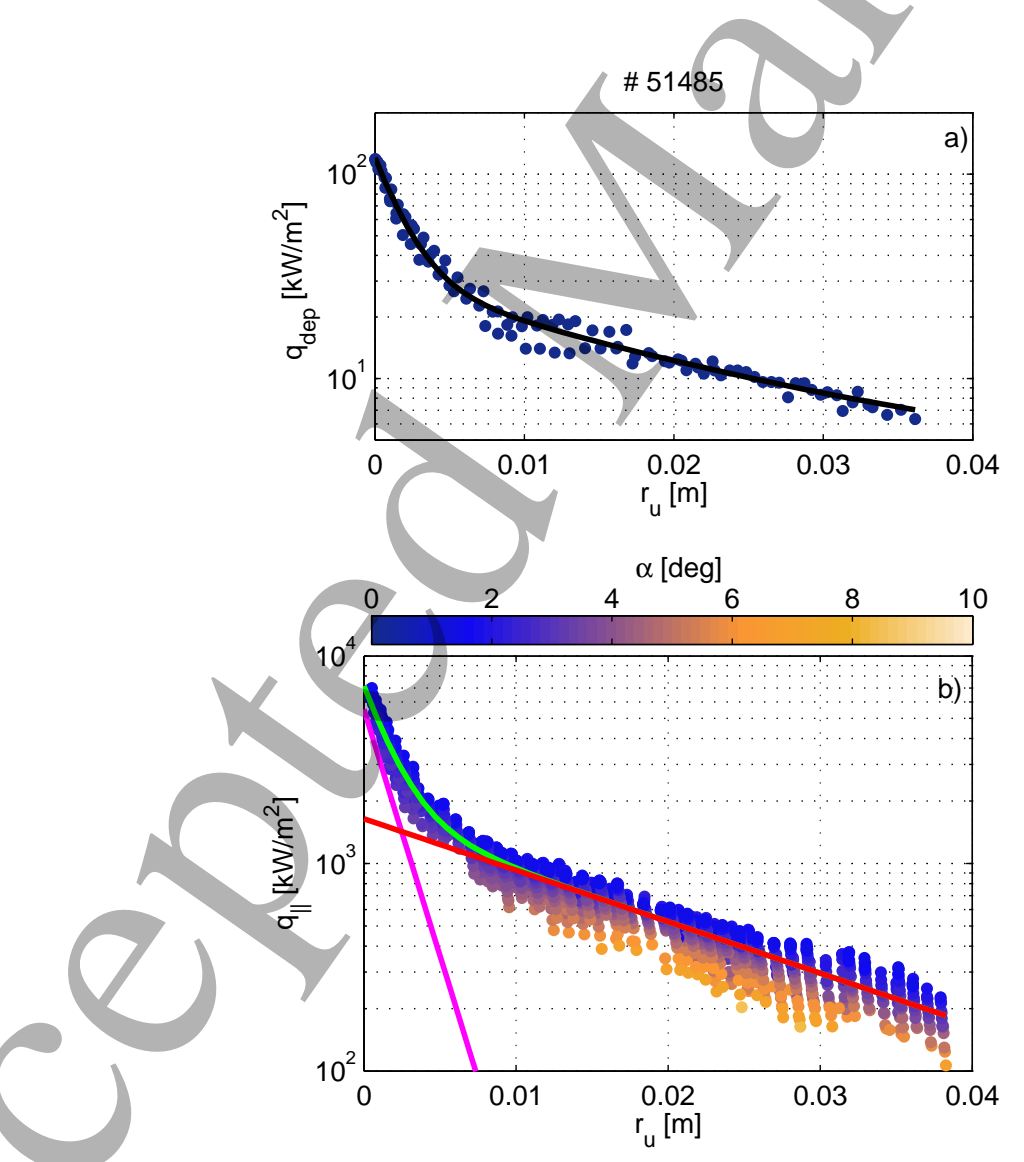

Figure 3: a) Cross-field heat flux at the limiter, $q_{\text {dep }}\left(r_{u}, \alpha=0\right)=q_{\perp}\left(r_{u}\right)+q_{B G}$, fitted with Eqs. (4, 5) (black line). b) Parallel heat flux profile $q_{\|}\left(r_{u}\right)$, color coded with the incidence angle $\alpha$, fitted with Eq. (2) (green line). The heat flux associated with the near and far SOL, $q_{n} \exp \left(-r_{u} / \lambda_{n}\right)$ and $q_{f} \exp \left(-r_{u} / \lambda_{f}\right)$, are plotted in magenta and red, respectively. 
We model $q_{\perp}\left(r_{u}\right)$ as a sum of two exponentials:

$$
q_{\perp}\left(r_{u}\right)=q_{\perp n} \exp \left(-r_{u} / \lambda_{\perp n}\right)+q_{\perp f} \exp \left(-r_{u} / \lambda_{\perp f}\right),
$$

where $\lambda_{\perp n}, \lambda_{\perp f}$ are the perpendicular heat flux decay length in the near and far SOL, respectively, and $q_{\perp n}, q_{\perp f}$ are the associated perpendicular heat flux magnitudes. The background heat flux $q_{B G}$ and the perpendicular heat flux parameters $\lambda_{\perp n}, \lambda_{\perp f}, q_{\perp n}$, and $q_{\perp f}$, are evaluated by fitting the deposited heat flux for tangential incidence $\left(q_{\text {dep }}\right.$ on the $\alpha=0$ line, blue dots in Fig. 2) with Eqs. $(4,5)$. An example of the fitting result is shown in Fig. 3a.

A fit with the sum of a single exponential and a background component is also performed for all the discharges. The description of $q_{\perp}$ by a sum of two exponentials or a single one is chosen based on the goodness of fit, $R^{2}$. In the case a single exponential fit produces $R^{2}$ equal to that resulting from the double exponential fit, $q_{\perp}\left(r_{u}\right)$ is still modeled by Eq. 5, where $q_{\perp n}=0$. The perpendicular heat flux at the contact point, defined by $r_{u}=0$, is given by $q_{\perp \theta}=q_{\perp}\left(r_{u}=0\right)=q_{\perp n}+q_{\perp f}$. This can be as large as the $20 \%$ of the peak heat load on the limiter [4]. Therefore, the perpendicular component of the heat flux can not be neglected when determining the parallel heat flux.

In this analysis we make the conservative assumption that the perpendicular heat flux $q_{\perp}\left(r_{u}\right)$ does not depend on the angle of incidence $\alpha$, even though a local enhancement of perpendicular transport through the "funnel effect" [22] is possible for grazing angles $\alpha \sim 0^{\circ}$. This could lead to an overestimation of $q_{\perp}$ for $\alpha \geq 1^{\circ}$. Anyways, as further discussed in Sec. 4, the funnel effect plays at most a minor role in our experiments. The parallel heat flux radial profile at the outer midplane is computed inverting Eq. (3) and accounting for the flux expansion:

$$
q_{\|}\left(r_{u}\right)=\frac{q_{d e p}\left(r_{u}, \alpha\right)-q_{\perp}\left(r_{u}\right) \cos \alpha-q_{B G}}{\sin \alpha} \frac{B_{u}\left(r_{u}\right)}{B_{l i m}\left(r_{u}\right)},
$$

where $q_{\perp}\left(r_{u}\right)$ is given by Eq. (5), $B_{u}$ and $B_{l i m}$ are the total magnetic field intensities at the outer midplane (upstream) and at the limiter, respectively. An example of the resulting parallel heat flux radial profile is shown in Fig. 3b. This is, in general, well described by a sum of two exponentials (Eq. 2). The parameters $\lambda_{n}, \lambda_{f}, q_{n}$ and $q_{f}$ are determined by fitting the whole $q_{\|}\left(r_{u}\right)$ profile with Eq. 2, for all the points for which $\alpha>1^{\circ}$. The same procedure as for fitting $q_{\perp}\left(r_{u}\right)$ is applied: a fit with a single exponential is also performed. In the case when a double exponential does not provide a better fit of the data, $q_{\|}\left(r_{u}\right)$ is still modeled by Eq. 2, where $q_{n}=0$.

\section{Perpendicular heat flux at the contact point}

The perpendicular heat flux at the contact point is given by $q_{\perp 0}=q_{\perp n}+q_{\perp f}$, accounting up to the $20 \%$ of the peak heat flux deposited on the tile, occurring for $\alpha \sim 3^{\circ}$. This leads to a ratio of perpendicular to parallel transport at the limiter $q_{\perp} / q_{\|} \sim 1 \%$. The "funnel effect" [22], usually used to justify $q_{\perp} / q_{||}$of the order of $10 \%$, could play a minor role in locally enhancing the perpendicular transport.

The perpendicular heat flux decay length $\lambda_{\perp n}$ is typically a few millimeters, while $\lambda_{\perp f}$ measures a few centimeters. No satisfactory scaling with the plasma parameters could be found for the perpendicular heat flux decay lengths.

Conversely, a nonlinear regression provides an empirical scaling for $q_{\perp 0}$ that reads

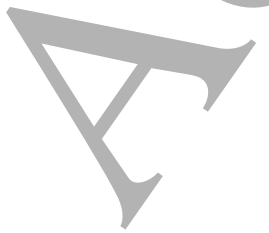

$$
q_{\perp, 0\left[k W / m^{2}\right]}=1.2 \cdot 10^{4} I_{p[M A]}^{2.25} \kappa^{-1.31} n_{e, a v\left[10^{19} m^{-3}\right]^{-0.50}} .
$$




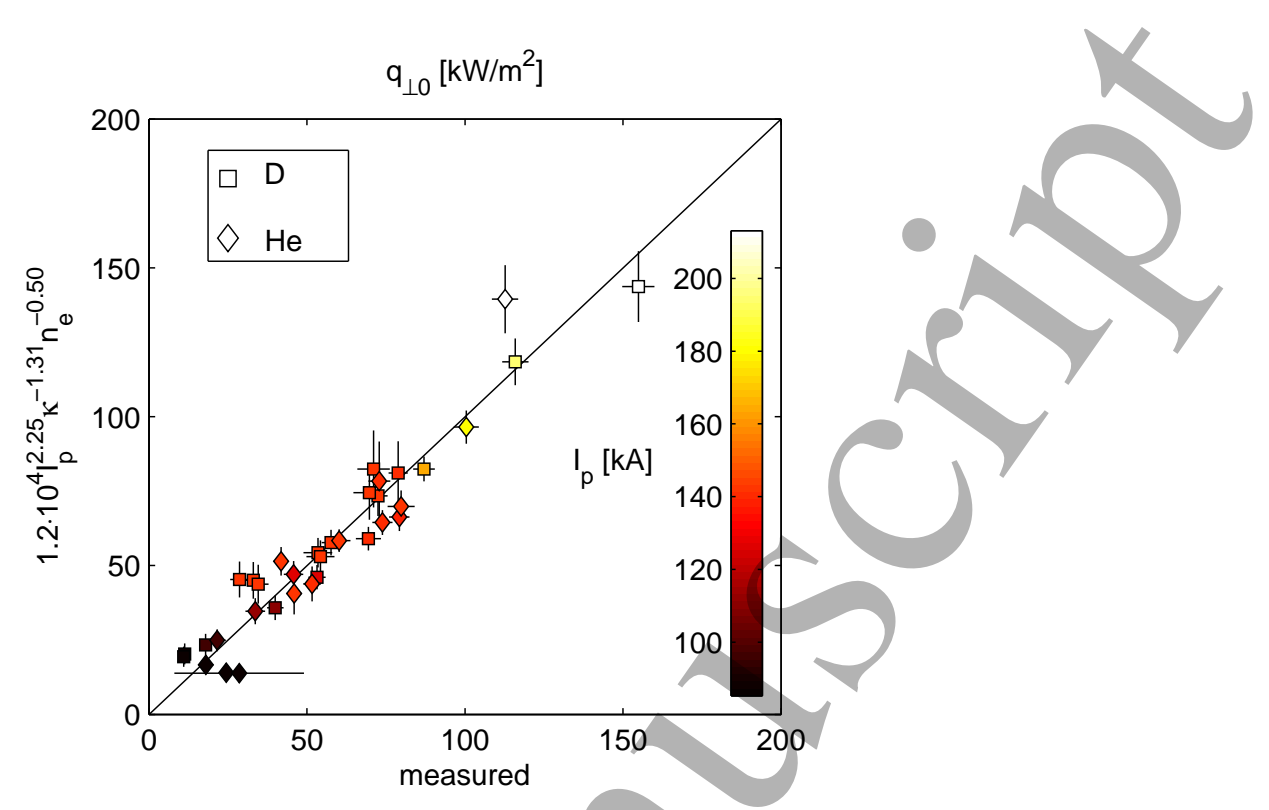

Figure 4: Empirical scaling of the perpendicular heat flux at the contact point $q_{\perp 0}$ with the plasma parameters, including D plasmas (squares) and He plasmas (diamonds). The points are color-coded with the plasma current.

The empirical scaling, including all the discharges presented in this paper (D, He), is shown in Fig. 4. The trends with the main plasma parameters are consistent with Ref. [4], where a similar scaling was determined using deuterium discharges only $\left(q_{\perp 0} \propto I_{p}^{2.5} \kappa^{-0.9} n_{e}^{-0.4}\right)$. The main trend is the increase of $q_{\perp 0}$ with the the square of the plasma current, which is consistent with an increase of the ohmic power $P_{\Omega}$. The decrease of $q_{\perp 0}$ with plasma density is consistent with a cooling of the SOL at the limiter at fixed power into the SOL, $P_{S O L}$, due to the conservation of total pressure along the field lines. The decrease of $q_{\perp 0}$ with the elongation $\kappa$ is consistent with the observed decrease of $P_{\Omega}$, due to the increase of confinement with elongation [23, 24] for constant plasma current $\left(I_{p}\right)$ and density, resulting in a lower power into the SOL, $P_{S O L}$.

In the following we compare the perpendicular heat flux at the contact point $q_{\perp 0}$ with the heat flux crossing the LCFS, $q_{L C F S}$. The power entering the SOL is evaluated as $P_{S O L}=P_{\Omega}-P_{\text {rad }}$, where $P_{\text {rad }}$ is computed from bolometric measurements. A nonlinear regression with the same plasma parameters as for the one in Eq, (7) provides an empirical scaling for $P_{S O L}$ :

$$
P_{S O L[k W]}=2.07 \cdot 10^{3} I_{p[M A]}^{1.32} \kappa^{-1.00} n_{e, a v\left[10^{19} m^{-3}\right]}^{0.05} .
$$

The weak dependence of $P_{S O L}$ on $n_{e, a v}$ is due to the fact that for increasing density $P_{\text {rad }}$ increases cooling the plasma, increasing in turns the resistivity i.e. $P_{\Omega}$ (for fixed $I_{p}$ ). We compute the heat flux crossing the separatrix as $q_{L C F S}=P_{S O L} / S_{L C F S}$, with $S_{L C F S}=4 \pi^{2} \sqrt{(\kappa) a_{m i n} R_{0}}$ the surface of the LCFS, being $a_{\text {min }} \approx 0.23 \mathrm{~m}$ and $R_{0} \approx 0.88 \mathrm{~m}$ the plasma minor and major radius respectively, having approximately the same value for all the discharges in the database. The ratio $q_{\perp 0} / q_{L C F S}$ scales therefore as

$$
q_{\perp 0} / q_{L C F S}=46.3 I_{p[M A]}^{0.93} \kappa^{0.19} n_{e, a v\left[10^{19} m^{-3}\right]}^{-0.45},
$$

i.e. it is approximately proportional to the plasma current and decreases with density, and depends weakly on elongation. 
Figure 5: Parallel heat flux radial profiles $q_{\|}\left(r_{u}\right)$ for the electron (blue dots) and ion (red dots) drift sides of the limiter, for the reference discharge \#51392. The fit with Eq. (2) are shown with solid lines. The fitting parameters $q_{n}, \lambda_{n}, q_{f}$ and $\lambda_{f}$ are displayed for the two cases.

\section{Near and far SOL parallel heat fluxes at the limiter}

For almost all of the investigated discharges, the parallel heat flux radial profile $q_{\|}\left(r_{u}\right)$ is well described by a sum of two exponentials (Eq. (2)), while their description with a single exponential as in the standard SOL model (Eq. (1)) is not equally satisfactory. The SOL can hence be divided into two regions, the "far" and "near" SOL respectively. We remark that the two tiles used for the analysis are located at the electron-drift side of the limiter. The parallel heat flux on the ion side of the limiter has been computed only for the reference discharge, \#51392. As a result, $q_{\|}\left(r_{u}\right)$ is well described by Eq. (2) on the ion-drift side of the limiter, too, as shown in Fig. 5. The values of the fitting parameters $\lambda_{n}, \lambda_{f}$ and $q_{f}$ are similar on both sides of the limiter. The near SOL heat flux magnitude $q_{n}$, though, is a factor of 2 lower on the ion drift side. This asymmetry in heat deposition could be due to poloidal asymmetries of the SOL driven by equilibrium poloidal flows, similarly to recent numerical simulations of the SOL [25]. In particular, as in [25], such an asymmetry is most likely to be due to the $\mathbf{E} \times \mathbf{B}$ drift, being mainly poloidal and directed towards the upper limiter for our discharges, shifting the stagnation point in the SOL from the outer mid plane towards the upper limiter.

The near SOL is observed to disappear in two cases for deuterium plasmas, namely for the lowest plasma current value investigated in the current scan $\left(I_{p}=85 \mathrm{kA}\right.$, the lowest value achievable on TCV for vertical stability, with $\left.n_{e, a v}=2.5 \cdot 10^{19} \mathrm{~m}^{-3}, \kappa=1.4, \delta=0\right)$ and for the highest density investigated in the density scan $\left(n_{e, a v}=4.7 \cdot 10^{19} \mathrm{~m}^{-3}\right.$, with $\left.I_{p}=140 \mathrm{kA}, \kappa=1.4, \delta=0\right)$. In Fig. 6 , the parallel heat flux radial profiles for the highest and lowest value of the plasma current (a), and for the highest and lowest value of the plasma density (b) are shown.

The variation of the fitting parameters used to describe the parallel heat fluxes $\left(\lambda_{n}, q_{n}, \lambda_{f}\right.$, and $q_{f}$ ) with the plasma current $I_{p}$, the plasma density $n_{e, a v}$ and the elongation $\kappa$ are displayed in Figures 7-10, respectively. In the figures, deuterium plasmas are plotted with blue squares, while the helium plasmas are shown with light blue diamonds, and the parameter range for which the 


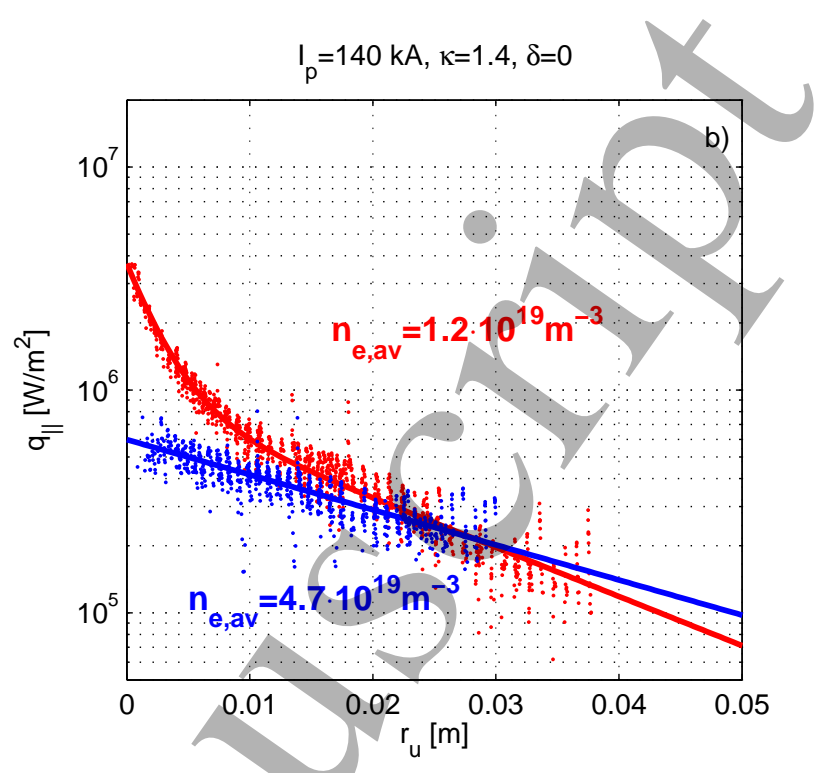

Figure 6: Disappearance of the near SOL at the limiter for low current and high density. Shown are the parallel heat flux radial profiles $q_{\|}\left(r_{u}\right)$ for a) $I_{p}=210 \mathrm{kA}$ (red dots) and $I_{p}=85 \mathrm{kA}$ (blue dots); b) $n_{e, a v}=1.2 \cdot 10^{19} \mathrm{~m}^{-3}$ (red dots) and $n_{e, a v}=4.7 \cdot 10^{19} \mathrm{~m}^{-3}$ (blue dots). The fit with Eq. (2) is shown with solid lines.

near SOL disappears in D plasmas is depicted with a green shaded region. The errorbars are given by the fit uncertainties. The main observed trends with respect to their dependence on the main plasma parameters $\left(I_{p}, n_{e, a v}, \kappa\right)$, displayed in the figures with dashed lines, are summarized in the following.

The near SOL width $\lambda_{n}$ is approximately constant over the explored range of currents (Fig. 7a), except for one point $\left(I_{p}=95 / \mathrm{kA}\right)$, for which the fitted $\lambda_{n}$ is close to the experimental spatial resolution. The near SOL vanishes for the lowest current value investigated $I_{p}=85 \mathrm{kA}$. This unexpected trend could result from two competing effects: in ohmic plasmas, increasing $I_{p}$, the safety factor decreases as $q_{e d g e} \propto 1 / I_{p}$ and the plasma temperature rises. Experiments including additional heating sources are advised for future works to disentangle the $q_{e d g e}$ and $T_{e}$ contributions to the trend of $\lambda_{n}$ with $I_{p}$. The near SOL width $\lambda_{n}$ decreases linearly with $n_{e, a v}$, eventually vanishing (Fig. 7b), and decreases linearly with $\kappa$ (Fig. 7c).

The near SOL parallel heat flux magnitude $q_{n}$ increases linearly with the plasma current $I_{p}$ (Fig. $8 \mathrm{a})$, consistently with the increase of the ohmic power, and it vanishes for low currents $\left(I_{p}=85\right.$ $\mathrm{kA}) \cdot q_{n}$ decrease for increasing density, after an initial increase (highlighted by a cubic interpolation in Fig. 8b), and vanishes for $n_{e, a v} \gtrsim 4.5 \cdot 10^{19} \mathrm{~m}^{-3} \cdot q_{n}$ decreases for increasing elongation (Fig. $8 \mathrm{c}$ ). This is consistent with the observed reduction of ohmic power with elongation at fixed plasma current and density, resulting in a lower power into the SOL, $P_{S O L}$.

The far SOL width $\lambda_{f}$ qualitatively agrees with the $\lambda_{q} \propto q_{\text {edge }} \propto 1 / I_{p}$ existing scaling [26] (dashed line in Fig. 9a). $\lambda_{f}$ increases linearly with $n_{e, a v}$ (Fig. 9b), consistently with previous observations in diverted plasmas [9], and decreases linearly with $\kappa$ (Fig. 9c), similarly to $\lambda_{n}$. This latter trend is unexpected since in existing scalings of the SOL width (e.g. [26]) usually $\lambda_{q} \propto q_{\text {edge }}$, where $q_{\text {edge }}$ is the safety factor at the LCFS. Since, for fixed plasma current and toroidal field, $q_{e d g e} \propto \kappa$, we would expect $\lambda_{q} \propto \kappa$, contrarily to our observations. Further investigations are required.

The far SOL parallel heat flux magnitude $q_{f}$ linearly increases with the plasma current (Fig. 10a), consistently with the increase of the ohmic power, linearly decreases with the plasma density $n_{e, a v}$ 

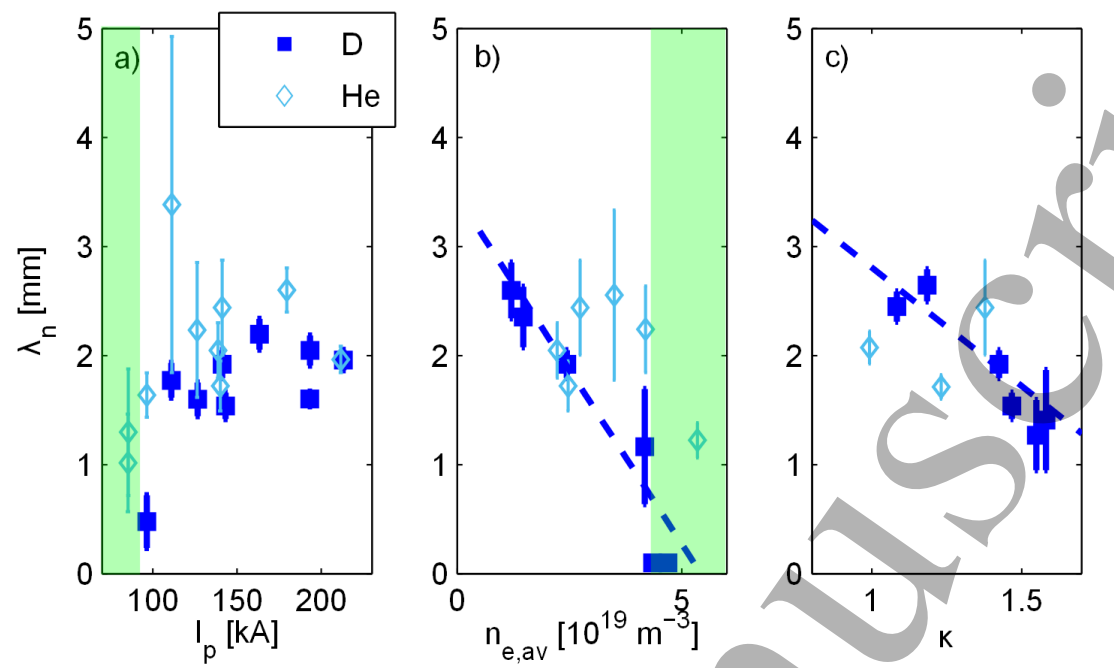

Figure 7: Variation of the near SOL width $\lambda_{n}$ with the plasma current $I_{p}$ (a), the line averaged density $n_{e, a v}(\mathrm{~b})$ and the elongation $\kappa(\mathrm{c})$, for both D (squares) and He (diamonds) plasmas. For all the discharges in a), $n_{e, a v}=2.5 \cdot 10^{19} \mathrm{~m}^{-3}, \kappa=1.4$, in b) $I_{p}=140 \mathrm{kA}, \kappa=1.4$, and in c) $I_{p}=140 \mathrm{kA}, n_{e, a v}=2.5 \cdot 10^{19} \mathrm{~m}^{-3}$. The region where the near SOL vanishes for $\mathrm{D}$ plasmas is shaded in green. Trends upon the plasma parameters are shown with dashed lines.
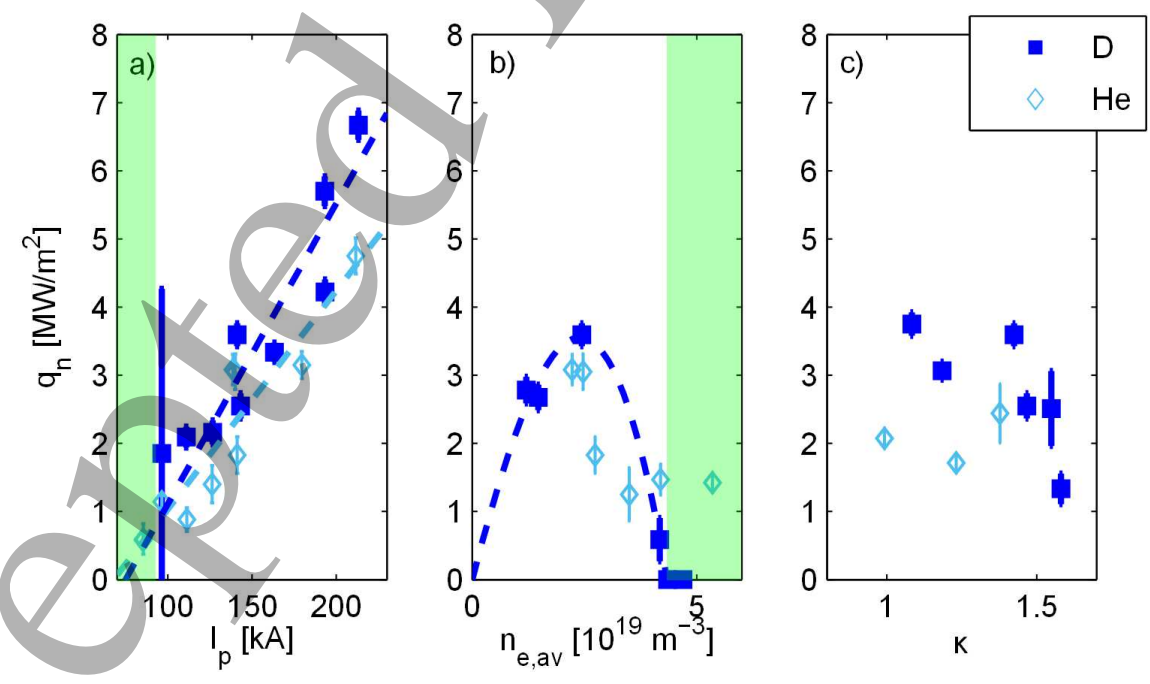

Figure 8: Variation of the near SOL heat flux magnitude $q_{n}$ with the plasma current $I_{p}$ (a), the line averaged density $n_{e, a v}$ (b) and the elongation $\kappa$ (c), for both D (squares) and He (diamonds) plasmas. For all the discharges in a), $n_{e, a v}=2.5 \cdot 10^{19} \mathrm{~m}^{-3}, \kappa=1.4$, in b) $I_{p}=140 \mathrm{kA}, \kappa=1.4$, and in c) $I_{p}=140 \mathrm{kA}, n_{e, a v}=2.5 \cdot 10^{19} \mathrm{~m}^{-3}$. The region where the near SOL vanishes for D plasmas is shaded in green. Trends upon the plasma parameters are shown with dashed lines. 

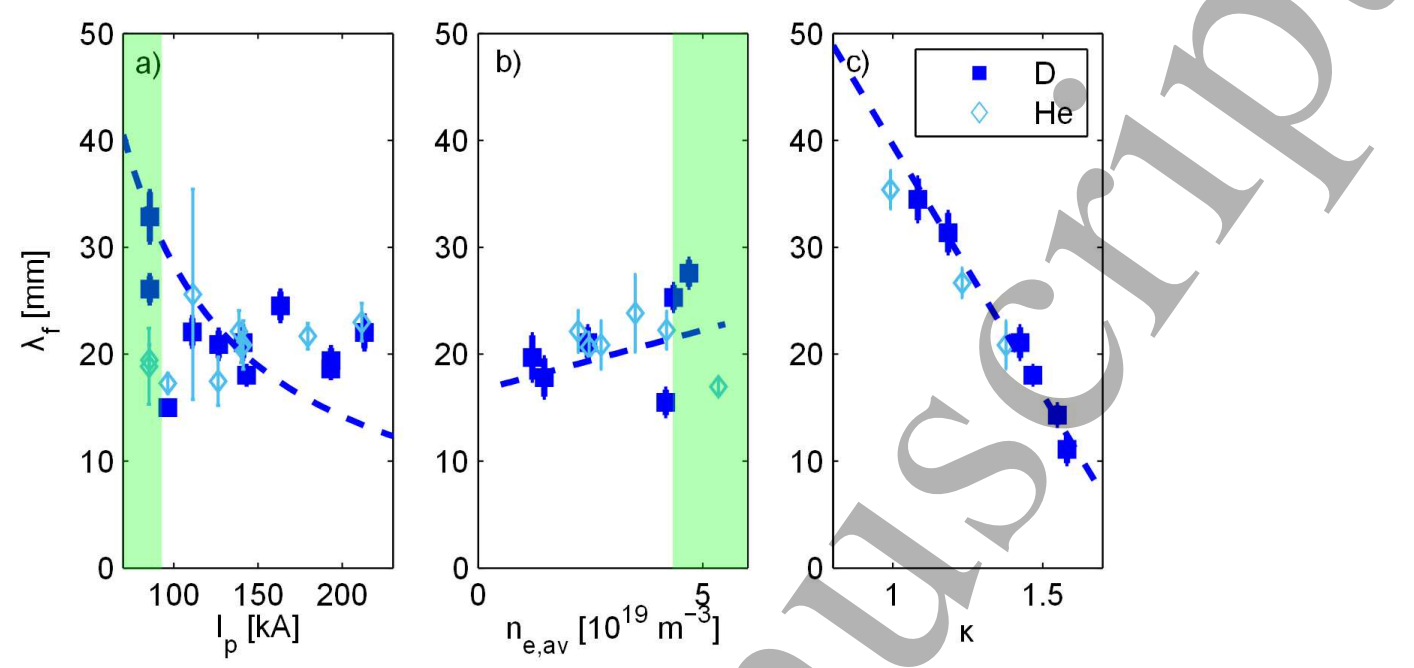

Figure 9: Variation of the far SOL width $\lambda_{f}$ with the plasma current $I_{p}(\mathrm{a})$, the line averaged density $n_{e, a v}$ (b) and the elongation $\kappa$ (c), for both D (squares)/and He (diamonds) plasmas. For all the discharges in a), $n_{e, a v}=2.5 \cdot 10^{19} \mathrm{~m}^{-3}, \kappa=1.4$, in b) $I_{p}=140 \mathrm{kA}, \kappa=1.4$, and in c) $I_{p}=140 \mathrm{kA}, n_{e, a v}=2.5 \cdot 10^{19} \mathrm{~m}^{-3}$. The region where the near SOL vanishes for D plasmas is shaded in green. Trends upon the plasma parameters are shown with dashed lines.
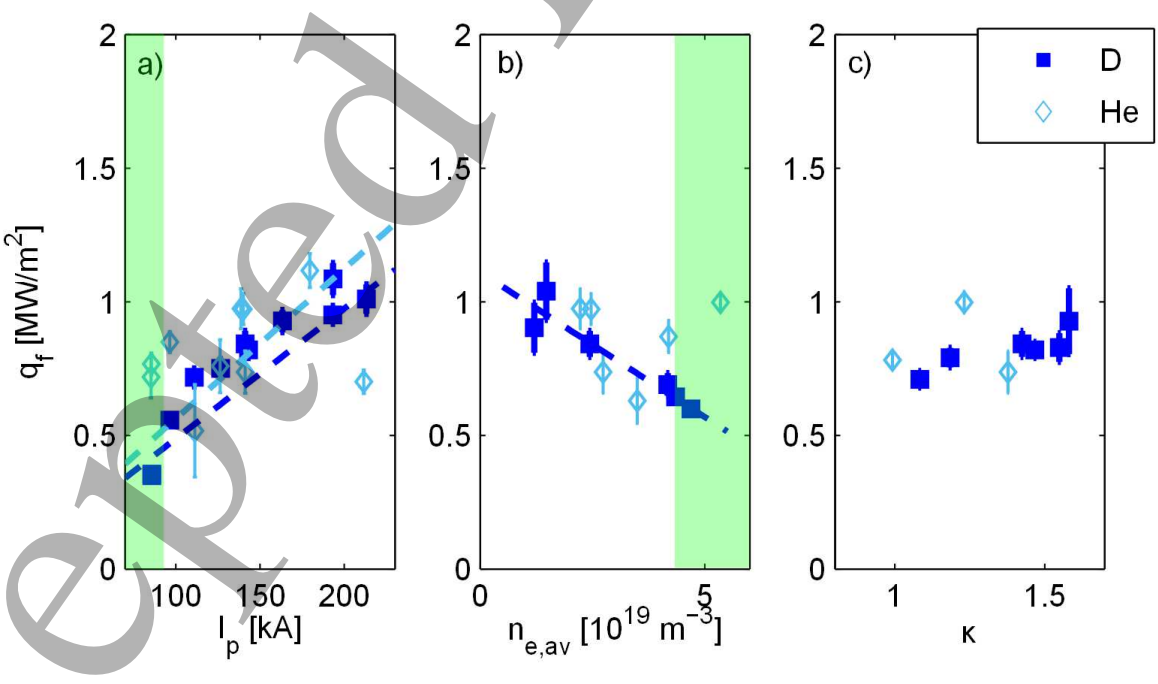

Figure 10: Variation of the far SOL heat flux magnitude $q_{f}$ with the plasma current $I_{p}$ (a), the line averaged density $n_{e, a v}$ (b) and the elongation $\kappa$ (c), for both $\mathrm{D}$ (squares) and He (diamonds) plasmas. For all the discharges in a), $n_{e, a v}=2.5 \cdot 10^{19} \mathrm{~m}^{-3}, \kappa=1.4$, in b) $I_{p}=140 \mathrm{kA}, \kappa=1.4$, and in c) $I_{p}=140 \mathrm{kA}, n_{e, a v}=2.5 \cdot 10^{19} \mathrm{~m}^{-3}$. The region where the near SOL vanishes for D plasmas is shaded in green. Trends upon the plasma parameters are shown with dashed lines. 


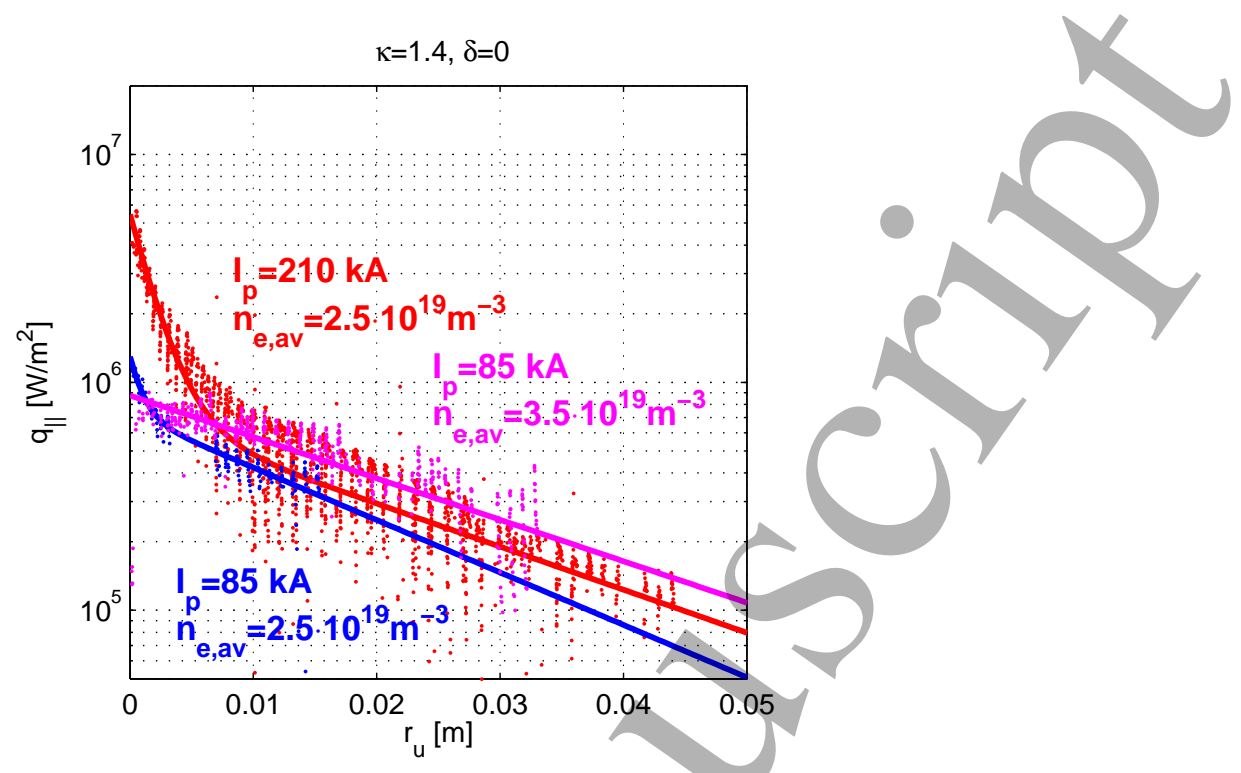

Figure 11: Parallel heat flux radial profiles $q_{\|}\left(r_{u}\right)$ in He for $I_{p}=210 \mathrm{kA}, n_{e, a v}=2.5 \cdot 10^{19} \mathrm{~m}^{-3}$ (red dots), $I_{p}=85 \mathrm{kA}, n_{e, a v}=2.5 \cdot 10^{19} \mathrm{~m}^{-3}$ (blue dots) and $I_{p}=85 \mathrm{kA}, n_{e, a v}=3.5 \cdot 10^{19} \mathrm{~m}^{-3}$ (magenta dots). The fit with Eq. (2) is shown with solid lines.

(Fig. 10b), and is approximately constant over the explored range of elongation $\kappa$ (Fig. 10c).

We report here the first observations of a near SOL in helium plasmas. Indeed, in He plasmas all the discharges except one exhibit a double scale length in the $q_{\|}\left(r_{u}\right)$ profiles, and the observed trends with the plasma parameters are similar to the D case. Nevertheless, there are some differences. Contrarily to the D case, the near SOL does not vanish for the lowest $I_{p}$ value $(85 \mathrm{kA})$, even though its strength $\left(\sim q_{n} \lambda_{n}\right.$, see Sec. 7) is decreased, as it is shown in Fig. 11. The near SOL does vanish for $I_{p}=85 \mathrm{kA}$ and an increased density $n_{e, a v}=3.5 \cdot 10^{19} \mathrm{~m}^{-3}$ (magenta profile in Fig. 11). The parallel heat flux profile for this discharge is well described by a single exponential.

The near SOL width $\lambda_{n}$ slightly decreases for $I_{p}<100 \mathrm{kA}$ (Fig. 7a) and does not vary with $n_{e, a v}$ (Fig. 7b), decreasing only for high density $\left(n_{e, a v}=5.3 \cdot 10^{19} \mathrm{~m}^{-3}\right)$. Contrarily to the $\mathrm{D}$ case, the near SOL is not observed to vanish for high densities. No clear trend of $\lambda_{n}$ with elongation is found (Fig. 7c).

The near SOL parallel heat flux magnitude $q_{n}$ decreases with elongation (Fig. 8c), similarly to what is observed for D plasmas, but its decrease starts at lower $\kappa$ in He.

The far SOL width $\lambda_{f}$ values are similar to the $\mathrm{D}$ case, but the scaling $\lambda_{q} \propto 1 / I_{p}$ [26] is not as satisfactory fulfilled as in the $\mathrm{D}$ case (Fig. 9a). $\lambda_{f}$ is approximately constant over the explored values of plasma current $I_{p}$. Also, $\lambda_{f}$ is constant rather than increasing with $n_{e, a v}$ (Fig. 9b).

\section{Scaling of the near and far SOL widths}

In Figure 12, we compare the SOL widths (near and far) resulting from the analysis of the parallel heat flux profiles from IR data, for both $\mathrm{D}$ and He discharges, with existing scalings derived from theoretical models. In particular, in Figure 12a, the far SOL width $\lambda_{f}$ is compared with the scaling proposed in Ref. [26], obtained from a quasi-linear (QL) model based on the gradient removal 

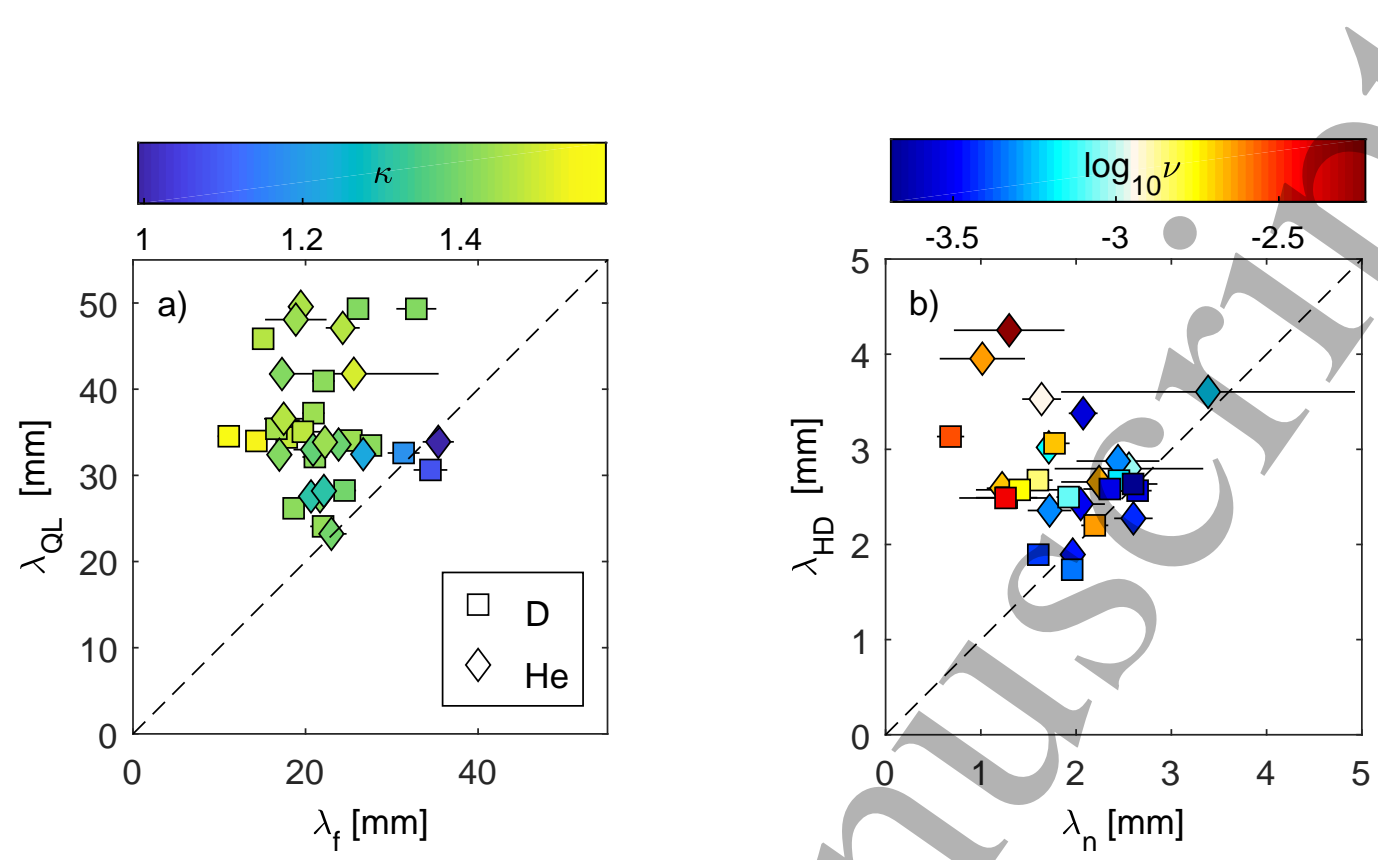

Figure 12: For both D (squares) and He (diamonds) plasmas: a) comparison of the experimental (from IR data) far SOL width $\lambda_{f}$ with the scaling given in Eq.) (10), color coded with the elongation $\kappa ; \mathrm{b}$ ) comparison of the experimental (from IR data) near SOL width $\lambda_{n}$ with the scaling given in Eq. (11), color coded with the normalized resistivity $\nu$.
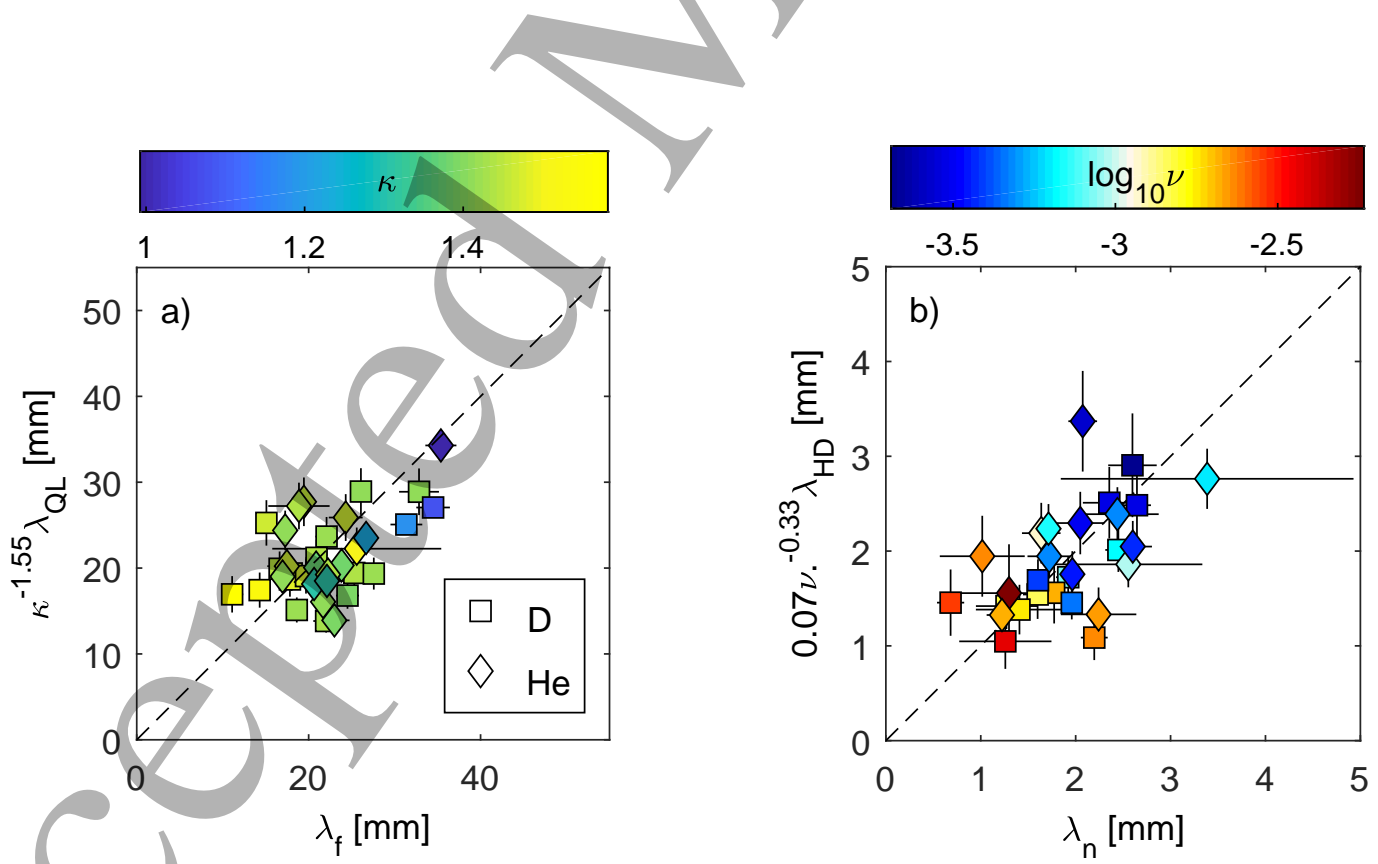

Figure 13: For both D (squares) and He (diamonds) plasmas: a) comparison of the experimental (from IR data) far SOL width $\lambda_{f}$ with the scaling given in Eq. (13), color coded with the elongation $\kappa ; \mathrm{b})$ comparison of the experimental (from IR data) near SOL width $\lambda_{n}$ with the scaling given in Eq. (14), color coded with the normalized resistivity $\nu$. 
theory [27], and successfully tested against the database presented in [28]. The scaling reads:

$$
\lambda_{Q L}[\mathrm{~m}]=4.96 \cdot 10^{-4} \times n_{e 0}^{0.07} T_{e 0}^{0.06} R_{0}^{0.68} q_{95}^{0.84} B_{\phi}^{-0.38},
$$

where $n_{e 0}, T_{e 0}$ are the plasma density and electron temperature at the LCFS, $R_{0}$ is the plasma major radius, $q_{95}$ is the edge safety factor, and $\lambda_{Q L}$ is the predicted SOL width. All quantities are expressed in SI units, and the temperature is in $\mathrm{eV}$ units. The scaling law is derived from a model for circular plasmas. Therefore we use for the comparison the cylindrical safety factor $q_{c y l}=q_{95} / \kappa$, i.e. the safety factor for a circular plasma, given only by its current and not by the shaping, instead of $q_{95}$. Furthermore, the model is developed to describe a "classical" SOL, for which only one scale length is considered.

As shown in Fig. 12a, there is an overall agreement between the experimental data and the scaling predictions. The scaling predicts successfully the far SOL width for circular plasmas $\kappa \sim 1$, but it overestimates the far SOL width for shaped plasmas $\kappa \gtrsim 1.4$. This has to be considered as the main cause of the discrepancies between the experimental and the predicted values. Also, as reported in Sec. 5, an increase in elongation could lead to an improved confinement of the core plasma, leading to less power crossing the LCFS and an overall narrower SOL.

Another possible cause for the observed discrepancies is the fact that the experimental data are measured at the HFS, while the scaling is developed for a poloidally averaged SOL width. The predicted values being larger than the experimental ones could therefore be interpreted as an HFS/LFS asymmetry, discussed in detail in Ref. [29]. This is consistent with the ballooning character of turbulent transport, and with recent numerical simulations of the SOL [30, 25].

In Fig. 12b, we compare the near SOL width $\lambda_{n}$ with the so-called Heuristic-Drift (HD) model [31]. This model has been originally developed for diverted H-mode plasmas. The main assumption is that, in this low-turbulence regime, the SOL width is determined by the competition of Bohm plasma flows towards the limiter plates $\left(v_{\|} \sim c_{s}\right)$ with cross field transport determined by the Pfirsch-Schluter currents, generated by the $\nabla B$ and curvature drifts. The resulting SOL width is predicted to be:

$$
\lambda_{H D}[\mathrm{~m}]=5671 \cdot P_{S O L}^{1 / 8} \frac{(1+\kappa)^{5 / 8} a^{17 / 8} B^{1 / 4}}{I_{p}^{9 / 8} R_{0}}\left(\frac{2 A}{Z^{2}(1+Z)}\right)^{7 / 16}\left(\frac{Z_{\text {eff }}+4}{5}\right)^{1 / 8},
$$

where $P_{S O L}$ is the power entering the SOL, $a$ is the plasma minor radius, $A$ and $Z$ are here, for simplicity, the mass and atomic number for the plasma ions, $A=2,4$ and $Z=1,2$ for $\mathrm{D}$, He plasmas, respectively. $Z_{e f f}$ is the plasma effective charge, taking into account the presence of impurities, and is adapted to match the plasma current using the ohmic and bootstrap current obtained from Ref. $[32,33]$, using $n_{e}$ and $T_{e}$ measurements from Thomson scattering, and assuming stationary state. All quantities are expressed in SI units. This scaling, despite having been derived for diverted H-mode, has been shown to describe satisfactorily the near SOL width for inboardlimited L-mode plasmas [34]. Still, experimental scalings for the SOL width in diverted H-mode, e.g. $\lambda_{q}[\mathrm{~mm}]=0.63 B_{\text {pol,u }}^{-1.19}[35,36]$, tend to overestimate $\lambda_{n}$ by a factor 3 .

As shown in Fig. 12b, there is indeed an overall good agreement between the experimental data and the HD scaling predictions. The main discrepancies could be attributed to the effect of both the plasma density and temperature, not included in Eq. (11). In the Figure, the points are color coded with the plasma normalized Spitzer resistivity $\nu$, combining both the variation of density and temperature. This is defined as [37]:

$$
\nu=\frac{e n_{e 0} R_{0} \eta_{\|}}{m_{i} c_{s 0}} \propto n_{e 0} T_{e 0}^{-2},
$$


where $e$ is the electron charge, $n_{e 0}, T_{e 0}$ and $c_{s 0}$ the plasma electron density, temperature, and the ion sound speed respectively, all evaluated at the LCFS, $m_{i}$ is the ion mass, $R_{0}$ is the plasma major radius (magnetic axis), and $\eta_{\|}$is the Spitzer resistivity [38]. The choice of the normalized resistivity here is driven by its effect on the near SOL presented in Ref. [4], and discussed in detail in the following section. For high values of $\nu$, the increased turbulence in the far SOL could gradually spread to the near SOL, and the hypothesis of absence of turbulence underlying the HD model would not be any longer satisfied.

We remark that the scaling in Eq. (11) is derived assuming that the electron magnetic drift determines the net transport. The equivalent scaling assuming predominant ion magnetic drift, $\lambda_{H D, i}=\lambda_{H D} Z^{-7 / 8}$, predicts smaller SOL widths for the He plasmas, while the prediction for D plasmas remain unvaried.

In the following, we propose empirical corrections to the QL and the HD scalings including the effects of elongation $\kappa$ and resistivity $\nu$, respectively. The evaluation of such corrections is performed through non-linear regressions over the database exposed beforehand. The refined scalings for the near and far SOL widths, respectively, read:

$$
\begin{aligned}
& \lambda_{f}=\kappa^{-1.55} \lambda_{Q L}, \\
& \lambda_{n}=0.07 \nu^{-0.33} \lambda_{H D}
\end{aligned}
$$

where $\lambda_{Q L}$ and $\lambda_{H D}$ are given in Eq. (10) using $q_{95}=q_{c y}$, and Eq. (11), respectively. The experimental data are compared with the refined scalings in Eqs. $(13,14)$ in Fig. 13, showing a better agreement.

\section{Power in the near SOL and correlation with resistivity and col- lisionality}

The heat fluxes associated with the near SOL can lead to the melting of the limiter [3], or to its damaging, and need therefore to be carefully taken into account in the design of future fusion reactors such as ITER [10]. What challenges the thermal limits of the first wall panels, rather than the near SOL width $\lambda_{n}$ or the associated heat flux magnitude $q_{n}$, is the combination of the two through the power entering the near SOL, $\Delta P_{S O L} \propto q_{n} \lambda_{n}$. Indeed, following Ref. [3], the power entering the SOL can be computed as

$$
P_{S O L}=2 \pi R_{L C F S} \frac{B_{\theta, u}}{B_{\phi, u}} \int_{0}^{\infty} 2 q_{\|}\left(r_{u}\right) d r_{u},
$$

where $R_{L C F S}$ is the major radius of the LCFS at the outer midplane, $B_{\theta, u}$ and $B_{\phi, u}$ are the poloidal and toroidal components, respectively, of the magnetic field at the outer midplane. $q_{\|}\left(r_{u}\right)$ is the parallel heat flux radial profile measured on one side of the limiter and remapped upstream. The factor two inside the integral accounts for the heat deposition on the two sides of the limiter (upper and lower), assumed to be equal.

Similarly, we define the power entering the near SOL as:

$$
\Delta P_{S O L}=4 \pi R_{L C F S} \frac{B_{\theta, u}}{B_{\phi, u}} \int_{0}^{\infty}\left[q_{\|}\left(r_{u}\right)-q_{\|, f}\left(r_{u}\right)\right] d r_{u}
$$

In Eq. 16, $q_{\|, f}\left(r_{u}\right)$ is obtained by extrapolating the parallel heat flux profile in the far SOL up to the LCFS. In the formalism used in this paper, $q_{\|, f}\left(r_{u}\right)=q_{f} \exp \left(-r_{u} / \lambda_{f}\right)$. The power entering the 


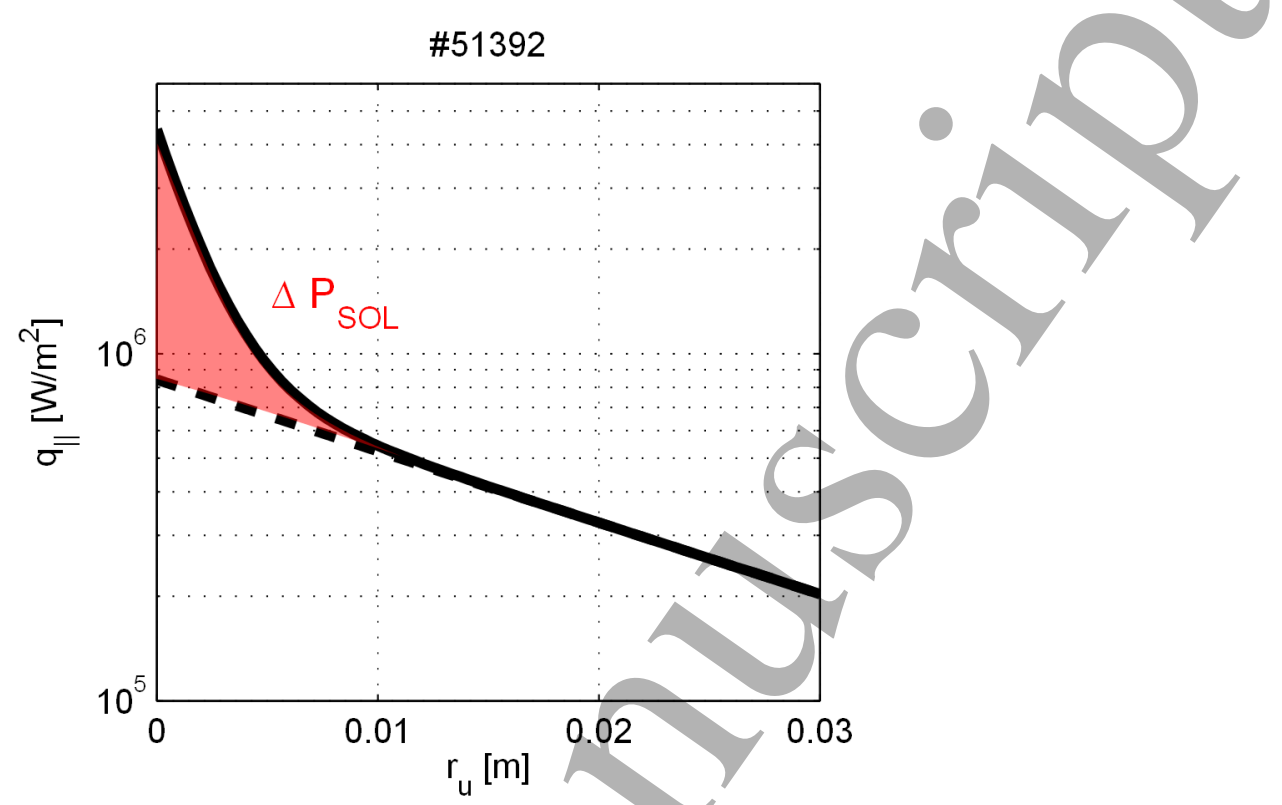

Figure 14: Schematics of the power entering the near SOL, $\Delta P_{S O L}$. The fit of Eq. (2) to the parallel heat flux radial profile $q_{\|}\left(r_{u}\right)$ is shown (solid line) for discharge \#51392. The dashed line represents the heat flux associated with the far SOL, $q_{f} \exp \left(-r_{u} / \lambda_{f}\right) . \Delta P_{S O L}$ corresponds to the integral of the red-shaded area.

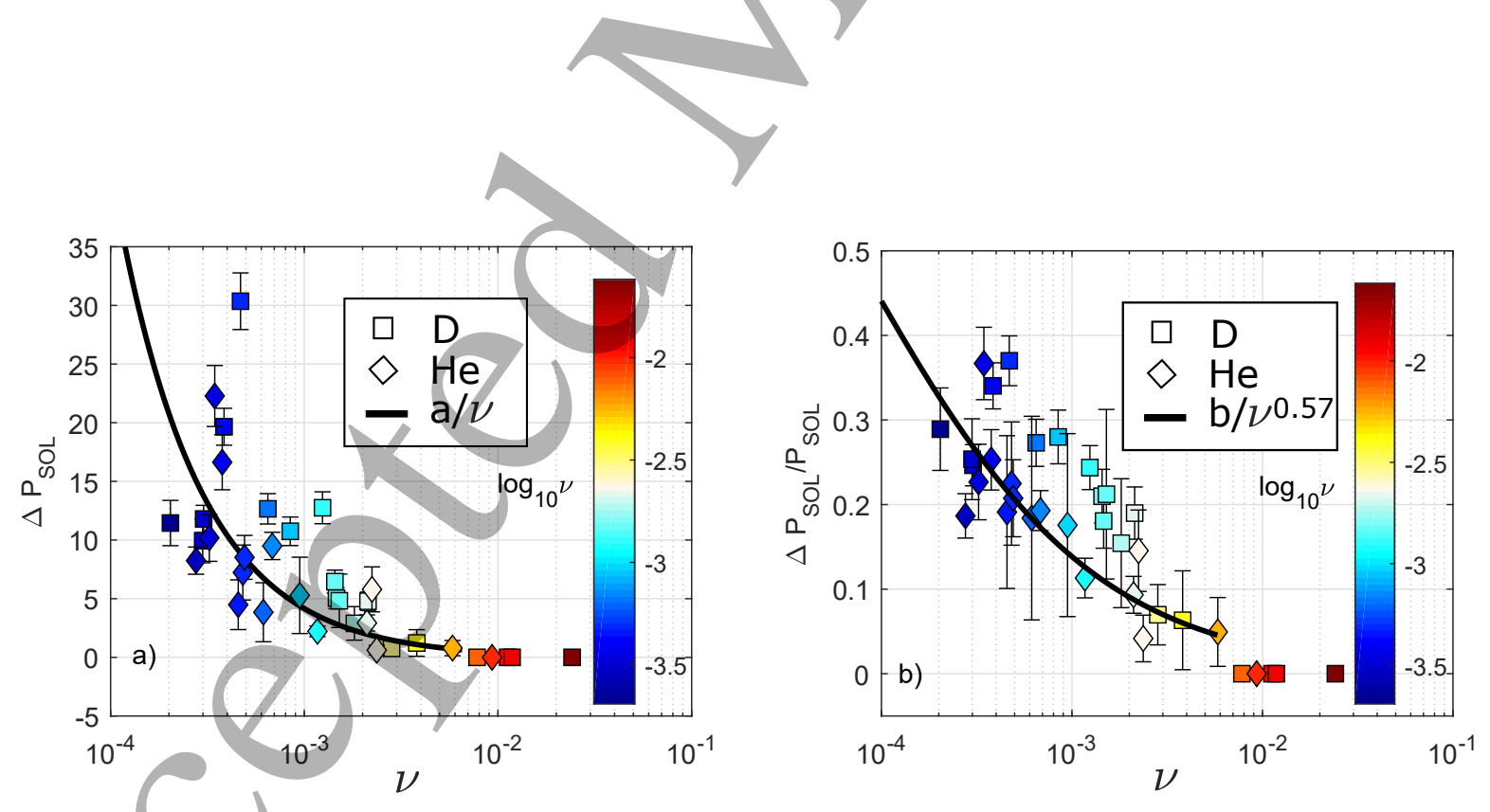

Figure 15: Power in the near SOL $\triangle P_{S O L}(\mathrm{a})$ and normalized to the total power in the SOL $P_{S O L}(\mathrm{~b})$ as a function of the normalized resistivity $\nu$, color coded with the logarithm of $\nu$. The results from the D (squares) and He (diamonds) experiments are plotted together with the lines $\Delta P_{S O L}=a / \nu$ (a) and $\triangle P_{S O L} / P_{S O L}=b / \nu^{0.57}(\mathrm{~b})$, plotted in black, where $a$ and $b$ are fitting parameters. 
near SOL is represented in Fig. 14 by the shaded area. Since the parallel heat flux radial profile $q_{\| \mid}\left(r_{u}\right)$ is described by a sum of two exponentials (Eq. (2)), the power entering the near SOL is given by

$$
\Delta P_{S O L}=4 \pi R_{L C F S} \frac{B_{\theta, u}}{B_{\phi, u}} \int_{0}^{\infty} q_{n} e^{-r_{u} / \lambda_{n}} d r_{u}=4 \pi R_{L C F S} \frac{B_{\theta, u}}{B_{\phi, u}} q_{n} \lambda_{n} .
$$

In the following, we use $\Delta P_{S O L}$ as a measure of the "strength" of the near SOL. In Ref. [4], a nonlinear regression with the plasma parameters of $\triangle P_{S O L}$ has been performed for previous deuterium experiments in TCV, showing that $\Delta P_{S O L}$ is well described by the empirical scaling

$$
\Delta P_{S O L[k W]}=192 T_{e, a v[k e V]}^{1.43} n_{e, a v\left[10^{19} m^{-3}\right]}^{-1.01} I_{p[M A]}^{-0.39} \kappa^{-0.76}|\delta|^{0.12},
$$

where $T_{e, a v}$ and $n_{e, a v}$ are the average plasma electron temperature and density, respectively. This empirical scaling has its main dependence on temperature and density, $\Delta P_{S O L} \propto T_{e}^{3 / 2} n_{e}^{-1}$, that can be approximated as $\Delta P_{S O L} \propto \nu^{-1}$, where $\nu$ is the normalized Spitzer resistivity ( Eq. (12)). The relationship $\Delta P_{S O L} \propto 1 / \nu$ is confirmed for all the present experiments, for both $\mathrm{D}$ and He plasmas. In Fig. 15a, $\Delta P_{S O L}$ is plotted for all the discharges against the normalized resistivity $\nu$. The curve $\Delta P_{S O L}(\nu) \propto 1 / \nu$ is plotted in black. The resistivity is here computed using the plasma electron density and temperature resulting from Thomson scattering measurements, averaged in the edge region $0.9<\rho<1$, where $\rho$ is the normalized poloidal flux coordinate ( $\rho=1$ at the LCFS). This method is preferred rather than using $n_{e}$ and $T_{e}$ measurements at the LCFS from the RP since this diagnostic was not available for all the discharges in the database. The resistivity used here provides an underestimate of the normalized resistivity defined in Eq. (12), since the temperature we used is larger than the temperature at the LCFS. As it is visible from the top panel of Fig. 15, a smooth transition occurs for high resistivities $\left(\nu \sim 0.7 \cdot 10^{-2}\right)$ to a regime where no near SOL heat flux feature is present. As shown in Fig. 15b, similar considerations hold for the fraction of power entering the near SOL $\Delta P_{S O L} / P_{S O L}$, transitioning to zero for $\nu>0.7 \cdot 10^{-2}$, and whose decrease with normalized resistivity is better described by $\Delta P_{S O L}(\nu) \propto 1 / \nu^{\gamma}$, where $\gamma=0.57$ is the best fitting parameter.

Almost identical trends could be found using, intstead of the normalized resistivity $\nu$, the SOL collisionality [2]

$$
\nu_{S O L}^{*}=L / \lambda_{e e}=10^{-16} n_{e 0}\left[\mathrm{~m}^{-3}\right] \frac{L[\mathrm{~m}]}{T_{e 0}[\mathrm{eV}]^{2}},
$$

where $L=2 \pi R_{0} q_{e d g e}$ is the connection length and $\lambda_{e e}$ is the electron-electron collisional mean free path. $n e 0$ and $T_{e 0}$ are the plasma electron density and temperature at the LCFS, respectively, but in this paper $\nu_{S O L}^{*}$ is evaluated using the edge values from Thomson scattering measurements, as it is done for the normalized resistivity. The normalized Spitzer resistivity $\nu$ and the SOL collisionality $\nu_{S O L}^{*}$ are clearly correlated, but $\nu_{S O L}^{*}$ provides some additional physical insight since it determines the transition between different regimes in the SOL [2]. In particular, a substantial decrease in $\Delta P_{S O L}$ is observed for $\nu_{S O L}^{*} \gtrsim 15$, corresponding to the transition to the conduction limited regime, where the electron temperature at the limiter can be sensibly lower than the temperature at the OMP. The transition to the near SOL-free regime happens for $\nu_{S O L}^{*} \sim 50$.

We remark that in these experiments, where the resistivity $\nu$ is varied only by changing the plasma current $I_{p}$ or the density $n_{e, a v}$, we could draw similar conclusions using the Greenwald fraction $f_{G}=n_{e, a v} / n_{G}=n_{e, a v}\left(\frac{I_{p}}{\pi a_{\min }^{2}}\right)^{-1}$, where $n_{G}$ is the Greenwald density and $a_{\min }$ is the plasma minor radius. It could hence be stated that $\Delta P_{S O L}$ decreases with $f_{G}$ and vanishes at sufficiently high Greenwald fractions $f_{G} \gtrsim 0.5$. Though, this would not be true in plasmas in which $\nu$ is changed by other means, e.g. modifying the plasma temperature by additional heating or impurity seeding. 


\section{Measurements of non-ambipolar currents and velocity shear at the limiter}

In the following, we discuss measurements of the floating potential $V_{f l}$ in TCV limited plasmas, performed at the limiter using flush mounted Langmuir probes (LP). The LP were operated in $I_{\text {sat }}$ and $V_{f l}$ mode (every other probe). A sweep in the plasma vertical position is performed to increase the spatial resolution of the measured profiles. A typical radial profile $V_{f l}$ is shown in Fig. 16. In the outer part of the far SOL, the floating potential is equal to the limiter potential $(V=0)$. Approaching the LCFS, $V_{f l}$ increases up to $V_{f l \text {,max }} \sim 10 \mathrm{~V}$. Entering the near SOL, the floating potential starts to decrease and changes sign, reaching large negative values at the contact point, $V_{f l, m i n} \sim-30 \mathrm{~V}$. A non-zero floating potential is the result of non-ambipolar currents flowing to the limiter, and the sign of the floating potential is given by the sign of the non-ambipolar currents. The presence of non-ambipolar currents in the near SOL of inboard limited plasmas has been reported in COMPASS [39]. In Ref. [39], the contribution of the non-ambipolar currents, measured both in the ion and electron-drift sides of the limiter, to the deposited heat fluxes has been found to be insufficient to explain the enhanced heat deposition in the near SOL. Similar conclusions have been drawn from recent nonlinear simulations of the TCV SOL [30].

In the following, we use the floating potential as a proxy for non-ambipolar currents. We parametrize the non-ambipolar currents with the floating potential drop in the SOL, that we define as

$$
\Delta V_{f l}=V_{f l, \max }-V_{f l, \min },
$$

where $V_{f l, \max }$ and $V_{f l, \min }$ are respectively the maximum and minimum value of $V_{f l}$ in the SOL, measured in the same side of the limiter. This is schematically illustrated in Fig. 16. An analo-

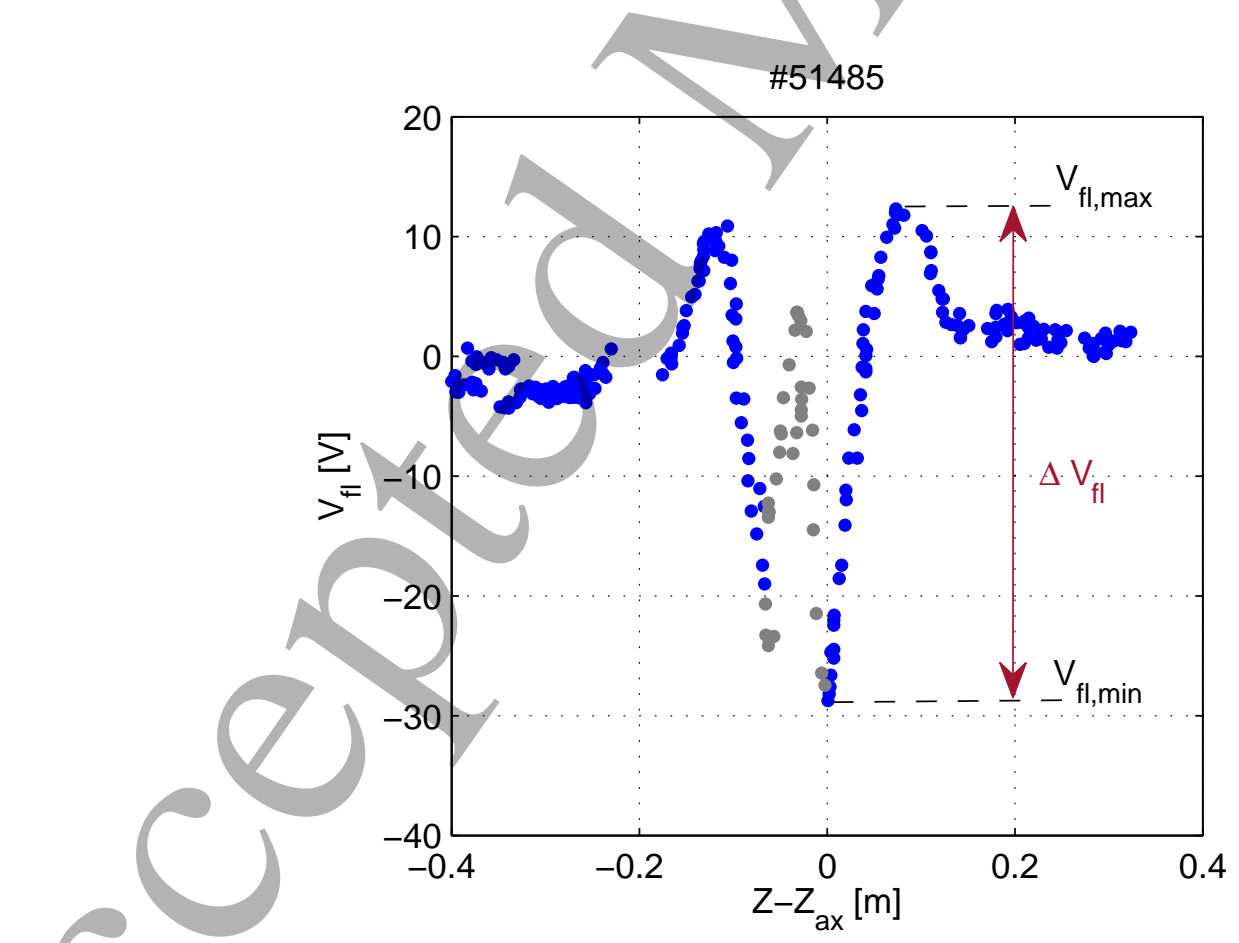

Figure 16: Floating potential profile along the vertical direction of the limiter for a typical discharge (blue dots). The grey dots identifies the region shadowed by the neighboring tile. The maximum and the minimum of the floating potential on the upper side of the limiter $V_{f l, \max }, V_{f l, m i n}$ and the potential drop in the SOL $\Delta V_{f l, \max }$ are indicated. 

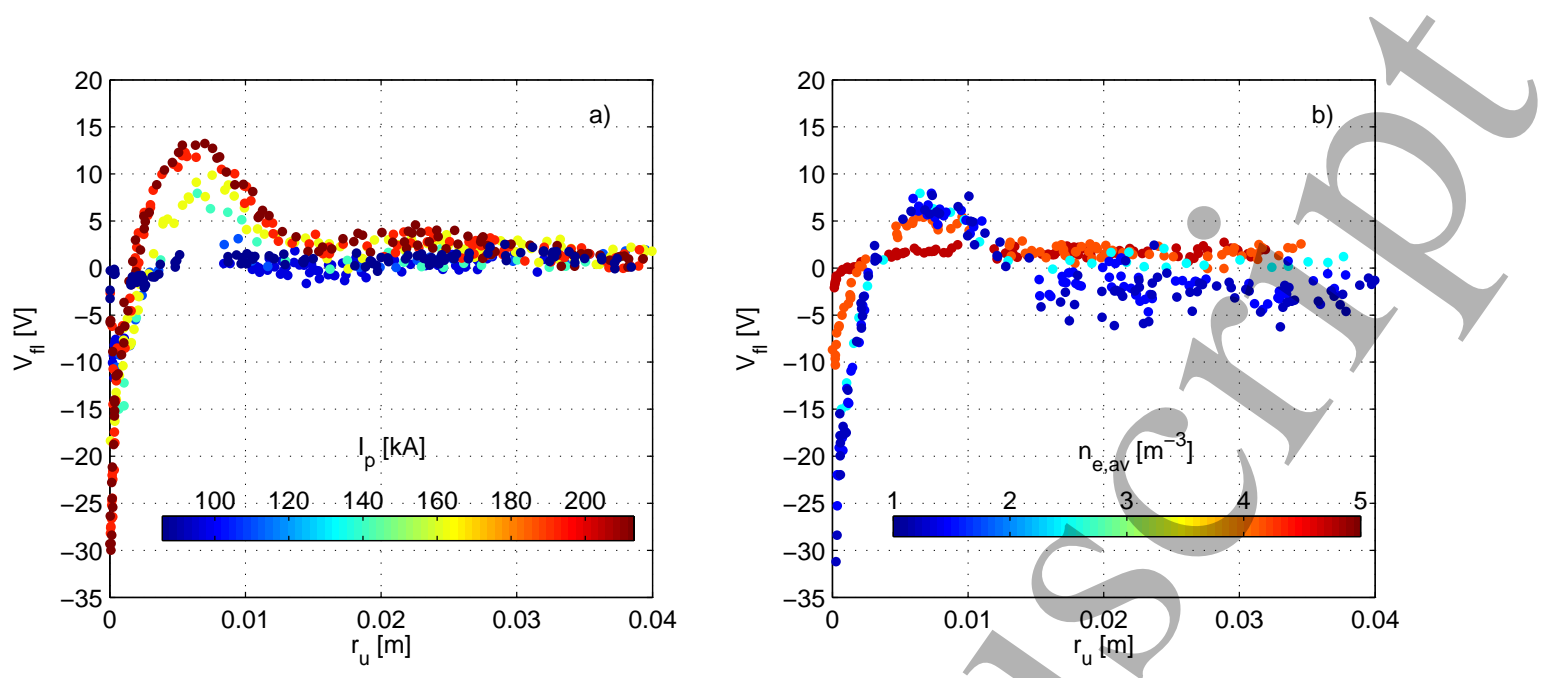

Figure 17: Floating potential profile remapped upstream $V_{f l}\left(r_{u}\right)$ for the $I_{p}$ scan (a), $\kappa=1.4, \delta=0$, $n_{e, a v}=2.5 \cdot 10^{19} \mathrm{~m}^{-3}$, and for the $n_{e, a v}$ scan (b), $I_{p}=140 \mathrm{kA}, \kappa=1.4, \delta=0$ (D plasmas).
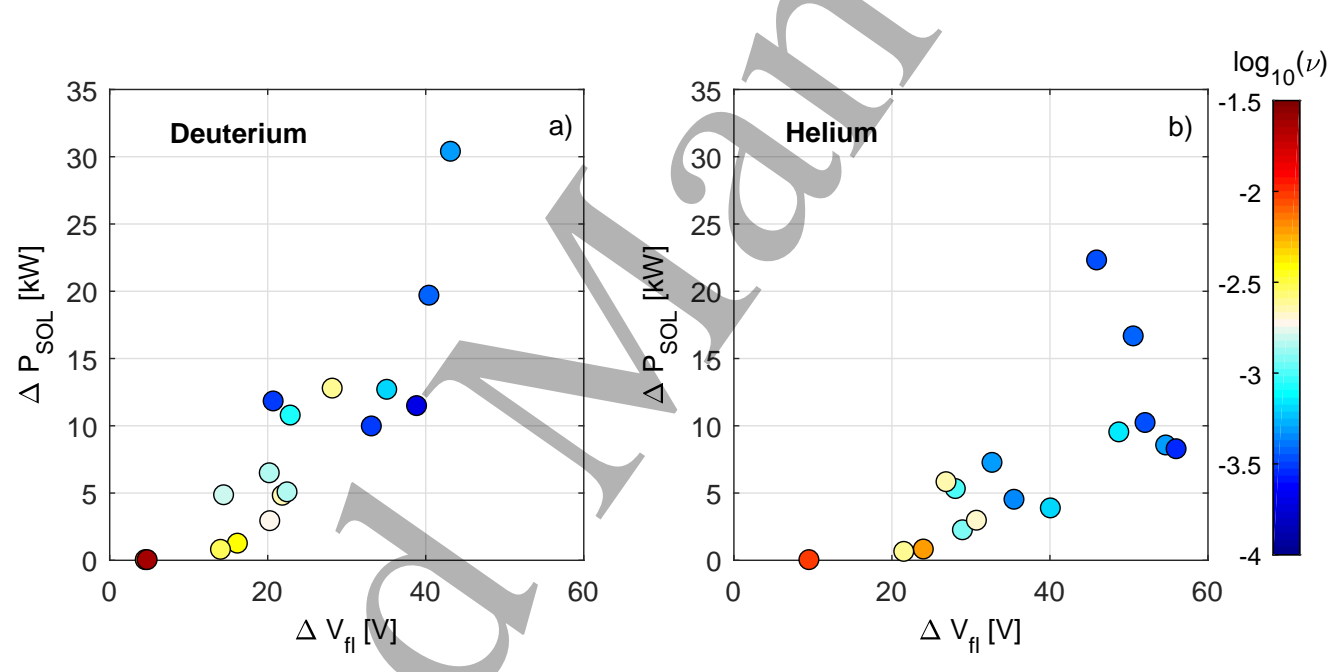

Figure 18: Power in the near SOL $\Delta P_{S O L}$ as a function of the floating potential drop $\Delta V_{f l}$, colorcoded with the normalized resistivity $\log _{10}(\nu)$, for D plasmas (a) and He plasmas (b).

gous floating potential drop in the SOL can be measured on both sides of the limiter, $\Delta V_{f l, i}$ and $\Delta V_{f l, e}$ for the ion and electron drift sides, respectively. No substantial asymmetry is observed in between the two sides of the limiter, being on average $\Delta V_{f l, i} / \Delta V_{f l, e}=1.13 \pm 0.24$ for $\mathrm{D}$ plasmas and $\Delta V_{f l, i} / \Delta V_{f l, e}=0.93 \pm 0.21$ for He plasmas. The electron drift side of the limiter (its upper part) is hence chosen for the following analysis (we identify $\Delta V_{f l} \equiv \Delta V_{f l, e}$ ) for consistency with what is done for the IR data analysis exposed in the previous sections.

In all the discharges discussed beforehand exhibiting a near SOL in the $q_{\|}\left(r_{u}\right)$ profiles, a floating potential drop in the near SOL has been measured. The variation of the floating potential radial profiles with the plasma current and density is shown for $\mathrm{D}$ plasmas in Fig. 17, where $V_{f l}\left(r_{u}\right)$ is plotted for different values of $I_{p}$ and $n_{e, a v}$. The intensity of the floating potential drop increases with $I_{p}$ and decreases with $n_{e, a v}$.

The non-ambipolar currents $\left(\Delta V_{f l}\right)$ are observed to almost vanish in the case where no near SOL steep gradient is measured in the parallel heat flux profiles, i.e. for the lowest current in the $I_{p}$ scan, 
$I_{p}=85 \mathrm{kA}$, and for the highest density in the density scan $n_{e, a v}=4.7 \cdot 10^{19} \mathrm{~m}^{-3}$.

The potential drop $\Delta V_{f l}$ is found to be correlated with the strength of the near SOL, $\Delta P_{S O L}$, as shown in Fig. 18, where $\Delta P_{S O L}$ is plotted against $\Delta V_{f l}$, color coded with the normalized resistivity $\nu$, for both D and He plasmas. For He plasmas, a larger $\Delta V_{f l}$, associated with increased non-ambipolar currents, is measured for the same power in the near SOL, $\triangle P_{S O L}$.

Even though non-ambipolar currents correlate with the power entering the near SOL, their presence alone is not sufficient to explain the enhancement in the heat deposition close to the limiter, consistently with previous experimental [39] and numerical [30] results. Lets consider, as an example, the typical discharge \#51485. The non-ambipolarity, resulting in a negative $V_{f l} \sim-30 \mathrm{~V}$ at the limiter, would lead to an increase of the sheath transmission factor $\gamma_{s h}$ of $\sim 20 \%$ with respect to the case of ambipolar conduction $\left(V_{f l}=0\right)$, according to Eq. 25.54 of ref. [2]. The enhancement of the parallel heat flux for the same discharge is more than one order of magnitude higher, $q_{n} / q_{f} \sim 6$. Another mechanism has therefore to come into play.

The potential drop $\Delta V_{f l}$ can be also considered as a measurement of the velocity shear in the near SOL. Indeed, the $\mathbf{E} \times \mathbf{B}$ drift velocity $\mathbf{v}_{E \times B}=\frac{\mathbf{E} \times \mathbf{B}}{B^{2}}$ in the SOL is mainly in the poloidal direction, since the electric field is mostly in the radial direction $\mathbf{E} \approx E_{r} \hat{r}$ and the magnetic field can be approximated by its toroidal component $\mathbf{B} \approx B_{\phi} \hat{\phi}$, where $\hat{r}$ and $\hat{\phi}$ are the unit vectors of the radial and toroidal coordinate, respectively. We can hence approximate $\mathbf{v}_{E \times B}$ with its poloidal component:

$$
\mathbf{v}_{E \times B}=\frac{\mathbf{E} \times \mathbf{B}}{B^{2}} \approx \frac{E_{r} \hat{r} \times B_{\phi} \hat{\phi}}{B^{2}} \approx-\frac{E_{r}}{B} \hat{\theta}=v_{\theta} \hat{\theta},
$$

where we approximated $B_{\phi} \approx B, \hat{\theta}$ is the unit vector of the poloidal coordinate, and $v_{\theta}=-E_{r} / B$ is the poloidal $\mathbf{E} \times \mathbf{B}$ velocity. The radial shear of the poloidal velocity is given by:

$$
v_{\theta}^{\prime} \equiv \frac{\partial v_{\theta}}{\partial r} \approx-\frac{1}{B} \frac{\partial E_{r}}{\partial r}=\frac{1}{B} \frac{\partial^{2} V_{p l}}{\partial r^{2}},
$$

where we neglected the dependence of $B$ upon the radial coordinate $r$, and we used the relationship $E_{r}=-\partial V_{p l} / \partial r$, with $V_{p l}$ being the plasma potential. The plasma potential is related to the floating potential by $V_{p l}=V_{f l}+\Lambda T_{e}$, with $\Lambda \sim 3$ for D plasmas. In the near SOL, we observe a strong variation of the floating potential on a scale length comparable with the near SOL parallel heat flux decay length $\lambda_{n}$. We assume that the radial gradient of the electron temperature is small with respect to the radial gradient of $V_{f l}$. Equation (22) can then be rewritten as:

$$
v_{\theta}^{\prime} \approx \frac{1}{B} \frac{\partial^{2} V_{f l}}{\partial r^{2}} \approx \frac{\Delta V_{f l}}{B \lambda_{n}^{2}} .
$$

The potential drop in the SOL $\Delta V_{f l}$ can hence be considered, in a first approximation, as a proxy for the velocity shear.

The results presented in Fig. 18 can then be interpreted in the following way: the presence of the near SOL $\triangle P_{S O L}$ is correlated with the presence of a radial shear in the $\mathbf{E} \times \mathbf{B}$ velocity. This interpretation is consistent with a recent theoretical model of the near SOL, presented in Ref. [40], and summarized in the following.

In the edge region (inside the LCFS), the radial electric field $E_{r}$ is always negative. Conversely, in the SOL the relationship $V_{p l} \sim 3 T_{e}$ holds, so the temperature profile of the typical form $T_{e}=T_{e 0} \exp \left(-r_{u} / \lambda_{T}\right)$ results in a positive $E_{r}$. The radial electric field has therefore to change sign across the LCFS, resulting in a shear of the poloidal $\mathbf{E} \times \mathbf{B}$ velocity. In the near SOL, the shearing rate $v_{\theta}^{\prime}$ is larger than the ballooning growth rate $\gamma_{b} \sim c_{s} / R_{0} \sqrt{2 R_{0} / L_{p}}\left(\gamma_{b} \sim 3.6 \cdot 10^{5} \mathrm{~s}^{-1}\right.$ 
for a typical TCV discharge), and the complex interaction of the turbulence with the sheath flows and the strong $\mathbf{E} \times \mathbf{B}$ shear results finally in the steepening of the pressure profile. The decrease of the $\mathbf{E} \times \mathbf{B}$ shear with collisionality, and the consequent suppression of the near SOL, is not yet portrayed in this model, and further analysis is needed to obtain the full theoretical description of this physical phenomenon.

\section{Comparison with the LFS measurements}

In all previous dedicated experiments $[3,4,6,7]$, the near SOL of inboard-limited plasmas had always been observed at the HFS. Still, no consensus could be reached on the presence of the near SOL on the LFS, being evident in C-Mod [7], not in DIII-D [6] and COMPASS [5], while no outboard measurements were available in TCV in Ref. [4], and the reciprocating probe in JET experiments [3] only probed the far SOL $\left(r_{u} \geq 20 \mathrm{~mm}\right)$.

In the following, we discuss the radial profiles of $q_{\| \mid}$and $V_{f l}$ at the outer midplane (OMP) and we compare them with those at the HFS that have been discussed earlier. The profiles at the OMP are obtained from reciprocating Langmuir probe (RP) measurements, and the methodology used is detailed in [29]. The $q_{\|}\left(r_{u}\right)$ (computed from $T_{e}$ and $I_{s a t}$ measurements) and $V_{f l}\left(r_{u}\right)$ profiles are plotted with red dots in Fig. 19a,b respectively for a typical discharge (\#51407). Similarly to the procedure adopted to parametrize the results obtained by IR thermography, the $q_{\|}\left(r_{u}\right)$ profile is fitted with a sum of two exponentials (Eq. 2). The result of the fit is plotted with solid lines in Fig. 19a, while the heat flux associated with the far SOL, $q_{f} \exp \left(-r_{u} / \lambda_{f}\right)$, is plotted with a black dashed line.

For discharge \#51407 (in red in Fig. 19), it is observed that the SOL is broader at the LFS than

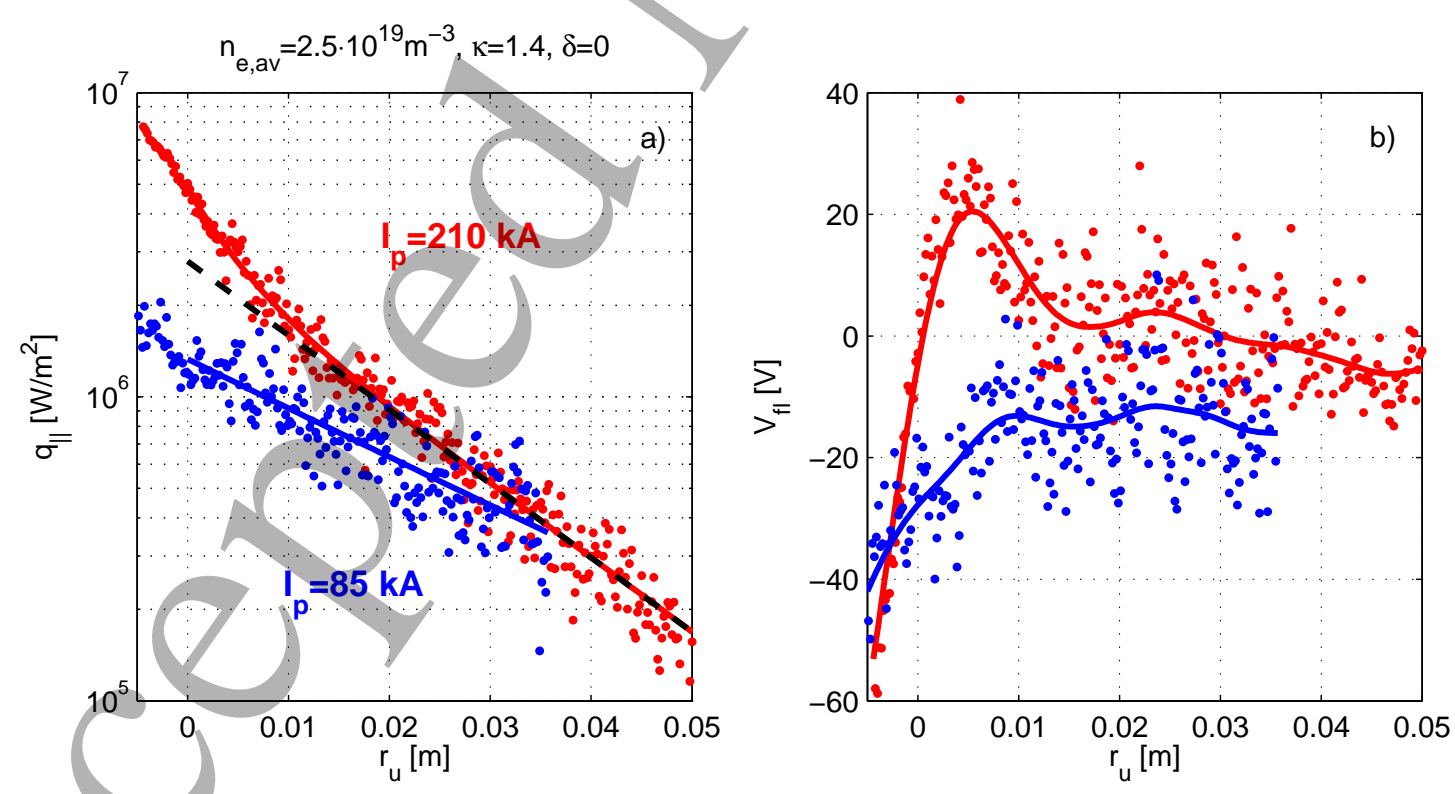

Figure 19: Radial profiles of parallel heat flux $q_{\|}\left(r_{u}\right)$ (a) and of floating potential $V_{f l}\left(r_{u}\right)$ (b) computed from RP data for the two same discharges as plotted in Fig. 6a, $I_{p}=210 \mathrm{kA}$ (red dots) and for $I_{p}=85 \mathrm{kA}$ (blue dots). a) The fits of $q_{\|}\left(r_{u}\right)$ with Eq. (2) are shown by solid lines, while the heat flux associated with the far SOL $q_{\|, f}\left(r_{u}\right)=q_{f} \exp \left(-r_{u} / \lambda_{f}\right)$ is shown with a black dashed line for the case $I_{p}=210 \mathrm{kA}$. b) The smoothed $V_{f l}\left(r_{u}\right)$ profiles are shown with solid lines. 
at the HFS, with $\lambda_{n, R P} / \lambda_{n, I R} \approx 3$ and $\lambda_{f, R P} / \lambda_{f, I R} \approx 2$. A broader SOL at the LFS is consistent with the ballooning character of turbulent transport, and with the poloidal asymmetries observed in numerical simulations of the SOL $[25,41,30]$. Also, the near SOL parallel heat flux is smaller at the LFS with respect to the HFS, being $q_{n, R P} / q_{n, I R} \approx 0.5$. Similar results hold for all the analyzed discharges. The asymmetries between the HFS and LFS during the plasma current scan in D discharges are investigated in detail in Ref. [29]. The extension of the quantitative analysis presented in [29] to the whole database here presented is reserved for future works.

In all cases where a near SOL is present in the HFS $q_{\|}\left(r_{u}\right)$ profile, it is also visible in the LFS $q_{\|}\left(r_{u}\right)$ profile. Also, the floating potential $V_{f l}$ becomes negative in the near SOL, as observed at the HFS from LP measurements. An example is shown in red in Fig. 19b. A velocity shear layer is then also present at the LFS midplane.

Furthermore, in the cases where the near SOL vanishes at the HFS, it also vanishes at the LFS. As an example, the $q_{\| \mid}\left(r_{u}\right)$ profiles from the RP for the two discharges with the highest and lowest value of $I_{p}$ (210 and $85 \mathrm{kA}$, respectively) are compared in Fig. 19a. The $q_{\|}\left(r_{u}\right)$ profiles measured at the limiter for the same two discharges have been shown previously in Fig. 6, where the near SOL is shown to vanish at the HFS for $I_{p}=85 \mathrm{kA}$. As shown in Fig. 19a, the near SOL vanishes at the LFS as well for the lowest current (blue profiles).

The vanishing of the near SOL in the $q_{\|}\left(r_{u}\right)$ profile is associated with a flattening of the $V_{f l}\left(r_{u}\right)$ profile at the HFS (see Fig. 17). On the LFS, the potential drop $\Delta V_{f l}$ is also reduced. Furthermore, the floating potential becomes negative through the entire SOL (blue profiles in Fig. 19b). This effect could be interpreted as a change in the poloidal distribution of the SOL currents, that are no longer flowing to the limiter plates and might be dissipated in the SOL.

\section{Conclusion}

Dedicated experiments have been performed in TCV to investigate the physics of the near SOL in inner wall limited discharges, in both $\mathrm{D}$ and He plasmas, scanning systematically the plasma current, density, and elongation. The parallel heat flux radial profiles, measured by means of IR thermography, show the presence of the near SOL for most discharges. For the first time, the near SOL is reported to disappear for high normalized resistivity $\nu \geq 0.7 \cdot 10^{-2}$, obtained here for low plasma current or high plasma density, for both $\mathrm{D}$ and He plasmas. The power in the near SOL is shown to scale as $\Delta P_{S O L} \propto 1 / \nu$. The far SOL width at the limiter is compared with the theoretical existing scaling from Ref. [26], finding good agreement for circular plasmas. An empirical correction with elongation is proposed. The near SOL width at the limiter agrees with the predictions from the Heuristic-Drift model [31] for low normalized resistivities. An empirical correction with $\nu$ is hence proposed. Non-ambipolar currents, correlated to the presence of a velocity shear, have been measured at the limiter with flush-mounted Langmuir probes. The power in the near SOL $\triangle P_{S O L}$ is shown to be correlated to the strength of the velocity shear which, according to a recent theoretical model [40], can affect the turbulence in the near SOL, locally steepening the pressure profiles. The parallel heat fluxes and the non-ambipolar currents measured at the limiter are compared with measurements taken at the Outer MidPlane (OMP) with a reciprocating probe. As a result, a near SOL is usually present at the OMP as well, but wider than at the limiter. The near SOL disappears together at the limiter and the OMP for high resistivities. Leveraging the findings here presented, a recipe to mitigate and suppress the near SOL heat fluxes during the start-up and ramp-down phases is currently being implemented and tested in TCV, and will be the subject of future publications. Also, the investigation of the near SOL in limited H-mode plasmas and the analysis of turbulence through blob dynamics using reciprocating Langmuir probe measurements are foreseen for future 
works.

\section{Acknowledgments}

This work has been carried out within the framework of the EUROfusion Consortium and has received funding from the Euratom research and training programme 2014-2018 under grant agreement No 633053, and from the Czech Science Foundation GA15-10723S. The views and opinions expressed herein do not necessarily reflect those of the European Commission and of the ITER Organization

\section{References}

[1] R.A. Pitts et al. Physics basis and design of the ITER plasma-facing components. Journal of Nuclear Materials, 415:S957-S964, 2011.

[2] P.C. Stangeby. The Plasma Boundary of Magnetic Fusion Devices . Institute of Physics Publishing Bristol and Philadelph, 2000.

[3] G. Arnoux et al. Scrape-off layer properties of ITER-like limiter start-up plasmas in JET. Nuclear Fusion, 53:073016, 2013.

[4] F. Nespoli et al. Heat loads in inboard-limited L-mode plasmas in TCV. Journal of Nuclear Materials, 463:393-396, 2015.

[5] J. Horacek et al. Narrow heat flux channels in the COMPASS limiter scrape-off layer. Journal of Nuclear Materials, 463:385-388, 2015.

[6] P.C. Stangeby et al. Power deposition on the DIII-D inner wall limiter. Journal of Nuclear Materials, 463:389-392, 2015.

[7] E.S. Marmar et al. Alcator C-Mod:/ research in support of ITER and steps beyond . Nuclear Fusion, 55:104020, 2015.

[8] B. LaBombard et al. Particle transport in the scrape-off layer and its relationship to discharge density limit in Alcator C-Mod . Physics of Plasmas, 8:2107, 2001.

[9] D. Carallero et al. An experimental investigation of the high-density transition of the scrape-off layer transport in ASDEX Upgrade. Nuclear Fusion, 54:123005, 2014.

[10] M. Kocan et al. Impact of a narrow limiter SOL heat flux channel on the ITER first wall panel shaping. Nuclear Fusion, 55:033019, 2015.

[11] A.R. Polevoi et al. Assessment of plasma parameters for the low activation phase of ITER operation. Nuclear Fusion, 53:123026, 2013.

[12] H. Meyer et al. Overview of progress in European Medium Sized Tokamaks towards an integrated plasma-edge/wall solution. Nuclear Fusion, 57:102014, 2017.

[13] S. Coda et al. Overview of the TCV Tokamak Program: Scientific Progress and Facility Upgrades. Nuclear Fusion, 57:102011, 2016. 
[14] F. Hofmann and G. Tonetti. Tokamak equilibrium reconstruction using Faraday rotation measurements. Nuclear Fusion, 28:1871-1878, 1988.

[15] F. Hofmann et al. Creation and control of variably shaped plasmas in TCV. Plasma Physics and Controlled Fusion, 36:B277-B287, 1994.

[16] J.A. Boedo et al. Fast scanning probe for the NSTX spherical tokamak. The Review of Scientific Instruments, 80:123506, 2009,.

[17] R. Maurizio et al. Infrared measurements of the heat flux spreading under variable divertor geometries in TCV. $43^{\text {rd }}$ EPS Conference on Plasma Physics, P4.027, 2016.

[18] A.G. McLean et al. A dual-band adaptor for infrared imaging - Review of Scientific Instruments, 83, 2012.

[19] A. Herrmann. Limitations for divertor heat flux calculations of fast events in tokamaks. ECA, 25A:2109-2112, 2001.

[20] U. Sheikh et al. A novel carbon coating technique for foil bolometers. Review of Scientific Instruments, 87, 2016.

[21] R.A. Pitts et al. The design of central column protection tiles for the TCV tokamak. Nuclear Fusion, 39:1433-1449, 1999.

[22] P.C. Stangeby C.S. Pitcher and J.D. Elder. The nature of plasma fluxes to surfaces nearly tangential to the magnetic field. Nuclear Fusion, 32:2079, 1992.

[23] H. Weisen et al. Effect of plasma shape on confinement and MHD behaviour in the TCV tokamak. Nuclear Fusion, 37:1741-1758, 1997.

[24] ITER Physics Basis. Chapter 2: Plasma confinement and transport. Nuclear Fusion, 39:2206, 1999.

[25] J. Loizu et al. Effect of the limiter position on the scrape-off layer width, radial electric field and intrinsic flows. Nuclear Fusion, 54:083033, 2014.

[26] F. D. Halpern et al. A theoretical interpretation of the main scrape-off layer heat-flux width scaling for tokamak inner-wall limited plasmas. Plasma Physics and Controlled Fusion, 58:084003, 2016.

[27] P. Ricci and B.N. Rogers. Plasma turbulence in the scrape-off layer of tokamak devices. Physics of Plasmas, 20:010702, 2013.

[28] J. Horacek et al. Multi-machine scaling of the main SOL parallel heat flux in tokamak limiter plasmas. Plasma Physics and Controlled Fusion, 58:074005, 2016.

[29] C. Tsui et al. Poloidal asymmetry in the narrow heat flux feature in the TCV scrape-off layer. Physics of Plasmas, 24:062508, 2017.

[30] F. Nespoli et al. Non-linear simulations of the TCV Scrape-Off Layer. Nuclear Materials and Energy, http://dx.doi.org/10.1016/j.nme.2016.10.019, 2016.

[31] R.J. Goldston. Heuridtic drift-based model of the power scrape-off width in low-gaspuff H-mode tokamaks. Nuclear Fusion, 52:013009, 2012. 
[32] O. Sauter et al. Neoclassical conductivity and bootstrap current formulas for general axisymmetric equilibria and arbitrary collisionality regime. Physics of Plasmas, 6:2834, 1999 .

[33] O. Sauter et al. Erratum: Neoclassical conductivity and bootstrap current formulas for general axisymmetric equilibria and arbitrary collisionality regime. Physics of Plasmas, 9:5140, 2002.

[34] R.J. Goldston. Theoretical aspects and practical implications of the heuristic drift SOL model. Journal of Nuclear Materials, 463:397-400, 2015.

[35] T. Eich et al. Inter-ELM power decay length for JET and ASDEX Upgrade: measurement and comparison with the heuristic drift-based model. Physical Review Letters, 107:215001, 2011.

[36] T. Eich et al. Scaling of the tokamak near the scrape-off layer H-mode power width and implications for ITER. Nuclear Fusion, 53:093031, 2013.

[37] F.D. Halpern et al. The GBS code for tokamak scrape-off layer simulations. Journal of Computational Physics, 315:388-408, 2016.

[38] R.J. Goldston and P.H. Rutherford. Introduction to Plasma Physics. Bristol: Institute of Physics Publishing, 1997.

[39] R. Dejarnac et al. Understanding narrow SOL power flux component in COMPASS limiter plasmas by use of Langmuir probes. Journal of Nuclear Materials, 463:381-384, 2015 .

[40] F. D. Halpern and P. Ricci. Velocity shear, turbulent saturation, and steep plasma gradients in the scrape-off layer of inner-wall limited tokamaks. Nuclear Fusion, 57:034001, 2017.

[41] C. Colin et al. Impact of the plasma-wall contact position on edge turbulent transport and poloidal asymmetries in 3D global turbulence simulations. Journal of Nuclear Materials, 463, 2015. 